\title{
ALGEBRAIC EXTENSIONS OF DIFFERENCE FIELDS
}

\author{
BY \\ PETER EVANOVICH(1)
}

\begin{abstract}
An inversive difference field $\mathscr{K}$ is a field $K$ together with a finite number of automorphisms of $K$. This paper studies inversive extensions of inversive difference fields whose underlying field extensions are separable algebraic. The principal tool in our investigations is a Galois theory, first developed by A. E. Babbitt, Jr. for finite dimensional extensions of ordinary difference fields and extended in this work to partial difference field extensions whose underlying field extensions are infinite dimensional Galois. It is shown that if $\mathcal{L}$ is a finitely generated separable algebraic inversive extension of an inversive partial difference field $\mathscr{K}$ and the automorphisms of $\mathscr{K}$ commute on the underlying field of $\mathscr{K}$ then every inversive subextension of $\mathcal{L} / \mathscr{K}$ is finitely generated. For ordinary difference fields the paper makes a study of the structure of benign extensions, the group of difference automorphisms of a difference field extension, and two types of extensions which play a significant role in the study of difference algebra: monadic extensions (difference field extensions $\mathcal{L} \mathscr{K}$ having at most one difference isomorphism into any extension of $\mathscr{K}$ ) and incompatible extensions (extensions $\mathcal{L} / \mathscr{K}, \mathcal{W} / \mathscr{K}$ having no difference field compositum).
\end{abstract}

Introduction. In [1], A. E. Babbitt, Jr. shows that if $\mathcal{L}$ is a finitely generated normal separable algebraic inversive difference field extension of the inversive difference field $\mathscr{K}$ then there exist inversive difference fields $\mathscr{K} \subseteq \mathcal{L}_{0} \subseteq \mathcal{L}_{1}$ $\subseteq \ldots \subseteq \mathcal{L}_{n}=\mathcal{L}$ where $\mathcal{L}_{0} / \mathscr{K}$ is finite dimensional and $\mathcal{L}_{i+1} / \mathcal{L}_{i}, i=1, \ldots$, $n-1$, is benign (Definition 4.1). Using this decomposition Babbitt shows $\mathcal{L} / \mathscr{K}$ is compatible (Definition 5.1) with an extension $\mathcal{M} \mathscr{K}$ if and only if $\mathcal{L}_{0} / \mathscr{K}$ is compatible with $\mathcal{M} / \mathscr{K}$. Babbitt then developed a Galois theory for the extension $\mathcal{L}_{0} / \mathcal{K}$ to examine further the questions of compatibility.

The Galois theory is extended in this paper to partial difference fields and, using the Krull topology, to infinite dimensional extensions. We use the theory in $\$ 2$ to prove that if $\mathcal{L}$ is a finitely generated separable algebraic inversive extension of the inversive difference field $\mathscr{K}$ and the transforming operators of $\mathscr{K}$ commute on the underlying field of $\mathscr{K}$ then every inversive subextension of $\mathcal{L} / \mathcal{K}$ is finitely generated over $\mathcal{K}$.

The remainder of the paper deals with ordinary difference fields. Propositions 5.1 and 5.2 and Theorems 5.2, 5.3 and 5.5 are restatements or extensions of

Received by the editors February 17, 1972.

AMS (MOS) subject classifications (1970). Primary 12H10; Secondary 12F05, $12 \mathrm{Fl0.}$

Key words and phrases. Partial and ordinary difference fields, algebraic extensions, Galois groups of difference field extensions, finitely generated extensions, limit groups, benign extensions, universal compatibility, monadicity, difference Galois groups, core of the Galois group of a difference field extension.

(1) Presented as a dissertation for the degree of Doctor of Philosophy at Rutgers, the State University and written under the direction of Professor Richard M. Cohn. This research was supported in part by National Science Foundation Grant GP-8548. 
Babbitt's results. They are discussed here in light of the new proofs and techniques offered by the Galois theory. The most important of these is Theorem 5.2 which shows compatibility of an extension $\mathcal{L} / \mathcal{K}$ depends only on the finite dimensional subextensions of $\mathcal{L} / \mathscr{K}$. The proof given here makes no use of the Babbitt composition mentioned above or of benign extensions.

A concept of limit group is developed in $\$ 3$ and then used to examine the structure of benign extensions. Theorem 5.7 shows that the group of difference automorphisms of a finitely generated universally compatible extension has its composition series equivalent to the composition series of the limit groups of that extension. Finally, examples will be given to show monadicity does not imply universal compatibility and there exist nonbenign algebraic difference field extensions with trivial cores.

Notation and conventions. Henceforth all difference fields will be inversive; that is, a difference field $\mathscr{K}$ will consist of a field $K$, called the underlying field of $\mathscr{K}$, and a finite number of automorphisms, $\sigma_{1}, \ldots, \sigma_{n}$, of $K$, called the transforming operators of $\mathscr{K}$. We will write $\left(K ; \sigma_{1}, \ldots, \sigma_{n}\right)$ for $\mathscr{K}$. If $G$ is a group, $f_{1}, \ldots, f_{n}$ are automorphisms of $G$ and $N \subseteq G$ then $\cap f_{1}^{k_{1}} \ldots f_{n}^{k_{n}}(N)$ will denote $\bigcap\left\{f_{1}^{k_{1}} \ldots f_{n}^{k_{n}}(N) \mid k_{1}, \ldots, k_{n}\right.$ are integers $\}$. If $n=1$ we will denote $f_{1}^{k}(N)$ by $N_{k}$. If $\ldots G_{-1}, G_{0}, G_{1}, \ldots$ are topological groups $\times G_{k}$ will denote the (topological) direct product $\times_{k=-\infty}^{\infty} G_{k}$. $\subset$ denotes proper containment and $<$ strictly less than.

1. The Galois correspondence. Let $\mathcal{K}$ be a difference field with underlying field $K$ and transforming operators $\sigma_{1}, \ldots, \sigma_{n}$. Let $\mathcal{L}$, with underlying field $L$ and transforming operators $\sigma_{1}, \ldots, \sigma_{n}$, be a (difference field) extension of $\mathcal{K}$. Further, take $L$ to be a normal separable algebraic extension of $K$. Make the Galois group $G$ of $L / K$ into a topological group by giving it the Krull topology [2]. $G$ is compact, Hausdorff, and has a basis at the identity, $e$, consisting of the collection of invariant, open (and hence closed and of finite index in $G$ ) subgroups of $G$. Each automorphism $\sigma_{i}$ of $L$ induces an automorphism (topological) $f_{i}$ of $G$ as follows:

$$
f_{i}(g)=\sigma_{i}^{-1} g \sigma_{i}
$$

Definition 1.1. A subgroup $H$ of $G$ is $\left(f_{i_{1}}, \ldots, f_{i_{k}}\right)$-stable if $f_{i_{j}}$ for $j=1, \ldots, k$ is an automorphism of $H$. $H$ is called stable if $H$ is $\left(f_{1}, \ldots, f_{n}\right)$-stable.

For each subextension $M / K$ of $L / K$ let $G(M)$ denote the subgroup of $G$ consisting of all automorphisms which leave every element of $M$ fixed. For each subgroup $H$ of $G$ let $L(H)$ denote the fixed field of $H$.

The following are easily verified. First, if $H$ is a stable subgroup of $G$ then, for $i=1, \ldots, n, \sigma_{i}$ is an automorphism of $L(H)$. Second, if $M$, with underlying field $M$, is a subextension of $\mathcal{L} / \mathcal{K}$ then $G(M)$ is a stable subgroup of $G$. 
If $H$ is a stable subgroup of $G$ then $\mathcal{L}(H)$ will denote the subextension of $\mathcal{L} / \mathcal{K}$ with underlying field $L(H)$ and transforming operators $\sigma_{1}\left|L(H), \ldots, \sigma_{n}\right| L(H)$. If $\mathcal{} M$ is as in the preceding paragraph then $G(\propto M)$ will denote the stable subgroup $G(M)$.

If $H$ is a closed invariant stable subgroup of $G$ then each $f_{i}$ induces an automorphism (topological) $h_{i}$ on $G / H$ as follows: for $g \in G, h_{i}(g H)=f_{i}(g) H$. We will denote $h_{i}$ by $f_{i}$ if no confusion arises.

Theorem 1.1. Let $\mathcal{L}, \mathcal{K}, L, K, G, \sigma_{1}, \ldots, \sigma_{n}, f_{1}, \ldots, f_{n}$ be as above.

(a) The mapping $\mathcal{M} \rightarrow G(\mathcal{M})$ establishes a 1-1 correspondence between the set of (inversive) subextensions of $\mathcal{L} / \mathscr{K}$ and the closed stable subgroups of $G$. If $\mathcal{M}$ is a subextension of $\mathcal{L} / \mathcal{K}$ then $\mathcal{L}(G(\mathcal{M}))=\mathcal{M}$ and if $H$ is a closed stable subgroup of $G$ then $G(\mathcal{L}(H))=H$.

(b) Let $M$ be a subextension of $\mathcal{L} / \mathbb{K}$ with underlying field $M$ normal over $K$. If $G^{\prime}$ is the Galois group of $M / K$ and $g_{1}, \ldots, g_{n}$ the automorphisms induced on $G^{\prime}$ by $\sigma_{1}\left|M, \ldots, \sigma_{n}\right| M$ respectively then there is a natural isomorphism $\varphi: G / G(\subset M) \rightarrow G^{\prime}$ (of topological groups) such that for $i=1, \ldots, n, \varphi h_{i}=g_{i} \varphi$ where $h_{i}$ is the automorphism of $G / G(c M)$ induced by $f_{i}$.

2. Finitely generated extensions. Let $\mathcal{L}$ be a difference field extension of the difference field $\mathscr{K}, L, K$ the underlying fields of $\mathcal{L}, \mathscr{K}$ respectively, and $\sigma_{1}, \ldots, \sigma_{n}$ the transforming operators of $\mathcal{L}$.

Let $S$ be a subset of $L$. We denote by $\mathscr{K} \ll S \gg$ the intersection of all subextensions of $\mathcal{L} / \mathcal{K}$ whose underlying fields contain $K \cup S$. Let $\Sigma$ be the set of all products of integral powers of the $\sigma_{i}$ and $S^{\prime}=\{\tau(a) \mid \tau \in \Sigma, a \in S\}$. The underlying field of $\mathscr{K} \ll S \gg$ is $K\left(S^{\prime}\right)$. If $S=\left\{a^{(1)}, \ldots, a^{(n)}\right\}$ is finite we write $K \ll a^{(1)}, \ldots, a^{(n)} \gg$ for $\mathscr{K} \ll S \gg$.

Definition 2.1. $\mathcal{L}$ is finitely generated over $\mathscr{K}$ if there exists a finite subset $S$ of $L$ such that $\mathcal{L}=\mathscr{K} \ll S \gg$. If the underlying extension of $\mathcal{L} / \mathscr{K}$ is Galois with Galois group $G$ and induced automorphisms $f_{1}, \ldots, f_{n}$ we say $\left(G ; f_{1}, \ldots, f_{n}\right)$ or simply $G$ is of finite type if and only if $\mathcal{L} / \mathcal{K}$ is finitely generated.

Note that Definition 2.1 is not equivalent to the definition of a finitely generated difference field extension given by Cohn in [4]. $\mathcal{L} / \mathcal{K}$ is finitely generated in the sense of Definition 2.1 above if and only if it is the inversive closure of a finitely generated, in the sense of Cohn, difference field extension of K.

Now, suppose $L / K$ is an algebraic extension and $L^{*}$ is the normal closure of $L$ over $K$. For each $i=1, \ldots, n$ we can extend $\sigma_{i}$ to an automorphism $\tau_{i}$ of $L^{*}$. Denote by $\mathcal{L}^{*}$ the difference field with underlying field $L^{*}$ and transforming operators $\tau_{1}, \ldots, \tau_{n} . \mathcal{L}^{*}$ will be called a normal closure of $\mathcal{L} / \mathcal{K}$. If $\mathcal{L} / \mathcal{K}$ is finitely generated then $\mathcal{L}^{*} / \mathscr{K}$ is finitely generated.

Proposition 2.1. Let $\mathcal{L}$ be a difference field extension of the difference field $\mathscr{K}$ with underlying extension $L / K$ Galois, $G$ the Galois group of $L / K, f_{1}, \ldots, f_{n}$ the 
respective automorphisms induced on $G$ by the transforming operators $\sigma_{1}, \ldots, \sigma_{n}$ of $\mathcal{L}$, and $\Sigma$ the set of all products of integral powers of the $\sigma_{i}$. A subextension $\mathcal{N} / \mathcal{K}$ of $\mathcal{L} / \mathcal{K}$ is finitely generated if and only if there exists an open subgroup $N$ of $G$ such that $\cap\left\{f_{\tau}(N) \mid \tau \in \Sigma\right\}=G(\mathcal{M})$ where if $\tau=\sigma_{i_{1}}^{k_{1}} \ldots \sigma_{i_{j}}^{k_{j}}$ then $f_{\tau}=f_{i_{j}}^{k_{j}} \ldots f_{i_{1}}^{k_{1}}$. Further, if for each pair $(i, j)$ of integers with $1 \leq i \leq n$ and $1 \leq j \leq n$ there is a $g_{i j}$ in $G$ such that $\sigma_{i}^{-1} \sigma_{j}^{-1} \sigma_{i} \sigma_{j}=g_{i j}$ (i.e. the transforming operators $\sigma_{1}, \ldots, \sigma_{n}$ commute on $K)$ then $\mathcal{N} / \mathscr{K}$ is finitely generated if and only if there exists an open subgroup $N$ of $G$ such that $G(\mathcal{M})=\cap f_{1}^{k_{1}} \ldots f_{n}^{k_{n}}(N)$. If the underlying extension of $M / \mathscr{K}$ is normal then $N$ in both cases above can be chosen to be an invariant subgroup of $G$.

Proof. Suppose $\subset \mathcal{M} \mathscr{K}$ is finitely generated, say $\mathcal{M}=\mathscr{K} \ll S \gg$ where $S$ is some finite subset of $M$, the underlying field of $\mathcal{A}$. Using the notation of Theorem 1.1, let $A=G(K(S))$. $A$ is closed and since $[K(S): K]<\infty,(G: A)<\infty$. B $=\bigcap\left\{g^{-1} A g \mid g \in G\right\}$ is a closed invariant subgroup of finite index in $G$. Since $G$ is compact $B$ is open. Denote $G(\mathcal{M})$ by $H$.

$$
\begin{aligned}
H & =G(K(\bigcup\{K(\tau(S)) \mid \tau \in \Sigma\}))=\bigcap\{G(K(\tau(S))) \mid \tau \in \Sigma\} \\
& =\bigcap\left\{f_{\tau}(A) \mid \tau \in \Sigma\right\} \supseteq \bigcap\left\{f_{\tau}(B H) \mid \tau \in \Sigma\right\} \supseteq H .
\end{aligned}
$$

Hence, $N=B H$ is open in $G$ and $\cap\left\{f_{\tau}(N) \mid \tau \in \Sigma\right\}=H$. If $M / K$ is normal then $H$ is an invariant subgroup of $G$ and $N$ is invariant.

Now, suppose the transforming operators $\sigma_{i}, \sigma_{j}$ commute on $K$. If $\tau \in \Sigma$ then $\tau=\tau_{1} \ldots \tau_{m}$ where $\tau_{i}=\sigma_{k}^{ \pm 1}$ for some transforming operator $\sigma_{k}$. If $i \in\{1, \ldots, m$ - 1) there exists $g \in G$ such that $\tau_{i} \tau_{i+1}=\tau_{i+1} \tau_{i} g . \quad f_{\tau}(N)=f_{\tau}(B) H$ $=f_{\alpha}\left(\tau_{i+1}^{-1} \tau_{i}^{-1}\left(f_{\beta}(B)\right) \tau_{i} \tau_{i+1}\right) H$ where $\alpha=\tau_{1} \ldots \tau_{i-1}$ and $\beta=\tau_{i+1} \ldots \tau_{m}(\alpha=e$ or $\beta$ $=e$ if $i=1$ or $m-1$ respectively). Hence

$$
f_{\tau}(N)=f_{\alpha}\left(\tau_{i}^{-1} \tau_{i+1}^{-1} g^{-1} f_{\beta}(B) g \tau_{i+1} \tau_{i}\right) H=f_{\alpha}\left(\tau_{i}^{-1} \tau_{i+1}^{-1} f_{\beta}(B) \tau_{i+1} \tau_{i}\right)
$$

since $B$ and thus $f_{\beta}(B)$ are invariant. Thus $f_{\tau}(N)=f_{\alpha}(B) H=f_{\gamma}(N)$ where $\gamma$ $=\tau_{1} \ldots \tau_{i+1} \tau_{i} \ldots \tau_{m}$. It follows from the above that if $p$ is any permutation of the letters $1, \ldots, m$ then $f_{\tau}(N)=f_{\delta}(N)$ where $\delta=\tau_{p(1)} \ldots \tau_{p(m)}$. Thus, for $\tau \in \Sigma$ there exist integers $k_{1}, \ldots, k_{n}$ such that $f_{\tau}(N)=f_{1}^{k_{1}} \ldots f_{n}^{k_{n}}(N)$, and hence $H=$ $\cap\left\{f_{1}^{k_{1}} \ldots f_{n}^{k_{n}}(N) \mid k_{1}, \ldots, k_{n}\right.$ integers $\}$.

Conversely, if $N$ is an open subgroup of $G$ such that $\cap\left\{f_{\tau}(N) \mid \tau \in \Sigma\right\}=H$ then $(L(N): K)<\infty$, and since $H \subseteq N, L(N)=K(S)$ where $S$ is some finite subset of $M . M=L\left(\cap\left\{f_{\tau}(N) \mid \tau \in \Sigma\right\}\right)=K(\bigcup\{K(\tau(S)) \mid \tau \in \Sigma\})=K\left(S^{\prime}\right)$ where $S^{\prime}=\bigcup\{\tau(S) \mid \tau \in \Sigma\}$. Hence $\mathcal{M}=\mathcal{K} \ll S \gg$.

Proposition 2.2. Suppose $n>1$ and the transforming operators of $\mathcal{L}$ commute on $K$. If $N$ is an invariant subgroup of $G$ and $M=\bigcap\left\{f_{1}^{k_{1}} \ldots f_{n-1}^{k_{n-1}}(N) \mid k_{1}, \ldots\right.$, $k_{n-1}$ integers $\}$ then for each integer $k, f_{n}^{k}(M)$ is $\left(f_{1}, \ldots, f_{n-1}\right)$-stable. 
Note that $G$ is of finite type if and only if $G$ has an open invariant subgroup $N$ such that $e=\cap f_{1}^{k_{1}} \ldots f_{n}^{k_{n}}(N)$.

In the context of Proposition 2.1, if $E$ is a subgroup of $G,\langle E\rangle$ will denote the stable subgroup $\cap f_{1}^{k_{1}} \ldots f_{n}^{k_{n}}(E)$.

Lemma 2.1. Let $G$ be a compact topological group, $H_{1}, H_{2}, \ldots$ a decreasing sequence of closed subgroups of $G$ with $\cap_{k=1}^{\infty} H_{k}=H$, and let $U$ be an open subset of $G$ containing $H$. Then there exists a positive integer a such that if $k \geq a, H_{k} \subseteq U$.

Theorem 2.1. Let $\mathscr{K}$ be a difference field with underlying field $K$ and transforming operators $\sigma_{1}, \ldots, \sigma_{n}$ which commute on $K, \mathcal{L}$ an extension of $\mathscr{K}$ with underlying field $L$ separable algebraic over $K$. If $\mathcal{L} / \mathscr{K}$ is finitely generated then any subextension is finitely generated.

Proof. We can assume $L / K$ is normal for if $\mathcal{L}^{*}$ is a normal closure of $\mathcal{L} / \mathcal{K}$ then $\mathcal{L}^{*} / \mathscr{K}$ is finitely generated. Let $G$ be the Galois group of $L / K$ and $f_{1}, \ldots, f_{n}$ the automorphisms induced on $G$ by $\sigma_{1}, \ldots, \sigma_{n}$ respectively.

Before proceeding with the proof of Theorem 2.1 we will prove that Theorem 2.1 implies that if $G$ is of finite type it has the descending chain condition (dcc) for closed stable subgroups. If $\left\{T_{i}\right\}$ is a decreasing sequence of closed stable subgroups then $T=\cap T_{i}$ is a closed stable subgroup whose fixed field is a finitely generated extension of $\mathscr{K}$. Proposition 2.1 implies there exists an open subgroup $U$ of $G$ such that $\langle U\rangle=T$. Lemma 2.1 implies there exists an $i$ such that $T_{i} \subseteq U$. Since $T_{i}$ is stable $T \subseteq T_{i} \subseteq\langle U\rangle=T$. The proof of the converse of this implication can be found in the proof of Theorem 2.1, to which we now return.

We proceed by induction on $n$, the number of transforming operators. The proofs of the case $n=1$ and the induction step are carried out simultaneously.

Let $\mathcal{M}$ be a subextension of $\mathcal{L} / \mathscr{K}$ and $H=G(\circlearrowleft M)$ the closed stable subgroup of $G$ corresponding to $M$ under the Galois correspondence. There exists by Proposition 2.1 an open invariant subgroup $N$ of $G$ such that $\langle N\rangle=e$. Let $E=\cap\left\{f_{1}^{k_{1}} \ldots f_{n-1}^{k_{n-1}}(N) \mid k_{1}, \ldots, k_{n-1}\right.$ integers $\}(E=N$ if $n=1) . E$ is a closed invariant $\left(f_{1}, \ldots, f_{n-1}\right)$-stable subgroup of $G$ (Corollary 2.1). $L(E)$, the subextension of $L / K$ corresponding to $E$, is the underlying field of a difference field with transforming operators $\sigma_{1}, \ldots, \sigma_{n-1}$. It is finitely generated over $\mathcal{K}^{\prime}$, the difference field with underlying field $K$ and transforming operators $\sigma_{1}, \ldots, \sigma_{n-1}$, since $N$ is an open subgroup of $G$ with $\cap\left\{f_{1}^{k_{1}} \ldots f_{n-1}^{k_{n-1}}(N) \mid k_{1}, \ldots, k_{n-1}\right.$ integers $\}$ $=E$. By the inductive assumption $\left(G / E ; f_{1}, \ldots, f_{n-1}\right)$ has the dcc for closed stable subgroups. (In the case $n=1, E$ is an open subgroup of the compact group $G$. Hence $G / E$ is a finite group.)

For $j=1,2, \ldots$ let

$$
N^{(j)}=H\left[\cap\left\{f_{1}^{k_{1}} \ldots f_{n}^{k_{n}}(N) \mid-j \leq k_{i} \leq j, 1 \leq i \leq n\right\}\right]
$$


and $H^{(j)}=\left\langle N^{(j)}\right\rangle . H^{(0)}$ will denote $H$.

The topological space $G / H$ of left cosets of $H$ is compact, Hausdorff and has a basis $\left\{N^{(j)} / H\right\}$ at $e H=H$ since, as one can easily see from Lemma 2.1, $\left\{\cap\left\{f_{1}^{k_{1}} \ldots f_{n}^{k_{n}}(N) \mid-j \leq k_{i} \leq j\right\} \mid j=0,1, \ldots\right\}$ is a basis at $e$ for $G$ [8]. If $g \in G$ and $g \notin H$ there exists a nonnegative integer $j$ such that $G H \cap N^{(j)} H$ $=g H \cap N^{(j)}=\varnothing$. Hence $\bigcap_{j=0}^{\infty} N^{(j)}=H$ and $\bigcap_{j=0}^{\infty} H^{(j)}=H$.

For each pair of nonnegative integers, $j, l$ let

$$
H_{j, l}=H^{(j)} \cap \bigcap_{k=0}^{l} f_{n}^{k}(E)
$$

and

$$
F_{j, l}=H^{(j)} \cap \bigcap_{k=0}^{l} f_{n}^{-k}(E) .
$$

For each positive integer $j$ and each nonnegative integer $l$ the natural maps defined below are monomorphisms of groups and, in the cases where $n>1$, are continuous and commute with the automorphisms induced on these (topological) quotient groups by $f_{1}, \ldots, f_{n-1}$.

$$
\begin{gathered}
\varphi_{j, l}: H_{j+1, l} / H_{j+1, l+1} \rightarrow H_{j, l} / H_{j, l+1}, \\
\psi_{j, l}: F_{j+1, l} / F_{j+1, l+1} \rightarrow F_{j, l} / F_{j, l+1}, \\
\alpha_{j, l}: H_{0, l} / H_{0, l+1} \rightarrow H_{j, l} / H_{j, l+1}, \\
\beta_{j, l}: F_{0, l} / F_{0, l+1} \rightarrow F_{j, l} / F_{j, l+1} .
\end{gathered}
$$

$H_{1, l} / H_{1, l+1}$ is a closed $\left(f_{1}, \ldots, f_{n-1}\right)$-stable subgroup of $A=H^{(1)} / H_{1, l+1}$. $\left(A ; f_{1}, \ldots, f_{n-1}\right)$ is of finite type since $B=H^{(1)} \cap \bigcap_{k=0}^{+1} f_{n}^{k}(N)$ is an open subgroup of $H^{(1)}$ with $\cap\left\{f_{1}^{k_{1}} \ldots f_{n-1}^{k_{n-1}}(B) \mid k_{1}, \ldots, k_{n-1}\right.$ integers $\}=H_{1, l+1}$. It is easy to see that subgroups of a group of finite type are of finite type. Hence, for each nonnegative integer $l,\left(H_{1, l} / H_{1, l+1} ; f_{1}, \ldots, f_{n-1}\right)$ is of finite type and by the inductive assumption has the dcc for closed stable subgroups. (In the case $n=1$, $H_{1, l} / H_{1, l+1}$ is a finite group.) Similarly we can show that, for each nonnegative integer $l,\left(F_{1, l} / F_{1, l+1} ; f_{1}, \ldots, f_{n-1}\right)$ has the dcc for closed stable subgroups.

For each nonnegative integer $l,\left\{H_{j, l} / H_{j, l+1} \mid j=1,2, \ldots\right\}$ is a decreasing sequence of closed stable subgroups of $H_{0, l} / H_{0, l+1}$ (up to an isomorphism of the form $\left.\varphi_{0, l} \ldots \varphi_{j-1, l}\right)$. Hence by the dcc there exists for each $l$ a positive integer $j_{l}$ such that $\varphi_{j, l}$ is an isomorphism if $j \geq j_{l}$. We claim that if $j \geq j_{l}$ then $\alpha_{j, l}$ is an isomorphism. If not, then it is not epic, so $S=H_{0, l} H_{j, l+1} \subset H_{j, l} . S$ is a closed subgroup of $H_{j, l}$. Since $H_{j, l}$ is a normal topological space there exists an open subgroup $U$ of $H_{j, l}$ such that $U S \subset H_{j, l} \cdot\left\{H_{i, l} \mid i=j, j+1, \ldots\right\}$ is a decreasing sequence of closed subgroups of $H_{j, l}$ whose intersection is $H_{0, l} \subseteq U S$. By Lemma 2.1 there exists $i \geq j$ such that $H_{i, l} \subseteq U S$. Now $H_{i, l} H_{j, l+1} \subseteq U S \subset H_{j, l}$ contradicting the fact that $\varphi_{i, l} \ldots \varphi_{j, l}$ is an isomorphism. 
Similarly we can show that for each nonnegative integer $l$ there exists a positive integer $k_{l}$ such that $\psi_{j, l}$ and $\beta_{j, l}$ are isomorphisms if $j \geq k_{l}$.

For each positive integer $j$ and each nonnegative integer $l$ the mappings $\mu_{j, l}$ and $\nu_{j, l}$ defined below are monomorphisms of topological groups.

$$
\begin{aligned}
\mu_{j, l}: H_{j, l+1} / H_{j, l+2} & \rightarrow H_{j, l} / H_{j, l+1}, \\
g H_{j, l+2} & \mapsto f_{n}^{-1}(g) H_{j, l+1}, \\
v_{j, l}: F_{j, l+1} / F_{j, l+2} & \rightarrow F_{j, l} / F_{j, l+1}, \\
g F_{j, l+2} & \mapsto f_{n}(g) F_{j, l+1} .
\end{aligned}
$$

It is not necessarily true that $\mu_{j, l}$ and $\nu_{j, l}$ commute with the automorphism induced on the above factor groups by $f_{1}, \ldots, f_{n-1}$. However, it is easy to see, using Corollary 2.1, that the image of $H_{j, l+1} / H_{j, l+2}$ in $H_{j, 0} / H_{j, 1}$ under $\mu_{j, 0} \ldots \mu_{j, l}$ is stable under the automorphism induced on the factor groups by $f_{1}, \ldots, f_{n-1}$. Hence, for each positive integer $j,\left\{H_{j, l} / H_{j, l+1} \mid l=0,1, \ldots\right\}$ is a decreasing sequence of closed $\left(f_{1}, \ldots, f_{n-1}\right)$-stable subgroups (up to isomorphism) of $H_{j, 0} / H_{j, 1}$. (In the case $n=1$ the sequence is a decreasing sequence of subgroups of the finite group $H_{j, 0} / H_{j, 1}$.)

Similarly, one shows that for each positive integer $j$ the sequence $\left\{F_{j, l} / F_{j, l+1} \mid l\right.$ $=0,1, \ldots\}$ is a decreasing sequence of closed $\left(f_{1}, \ldots, f_{n-1}\right)$-stable subgroups of $F_{j, 0} / F_{j, 1}$.

Using the induction hypotheses, there exists for each positive integer $j$ a nonnegative integer $l(j)(m(j))$ such that if $l \geq l(j)(l \geq m(j))$ then $\mu_{j, l}\left(\nu_{j, l}\right)$ is an isomorphism.

Up to an isomorphism of the form $\varphi_{1,0} \ldots \varphi_{1, j-1} \mu_{j, 1} \ldots \mu_{j, l}$ we can consider $\left\{H_{j, l} / H_{j, l+1} \mid j, l+1\right.$ positive integers $\}$ as a collection of closed stable subgroups of $H_{1,0} / H_{1,1}$. Also if $j \geq k$ and $l \geq m$ we may consider, up to an isomorphism of the form $\varphi_{k, m} \ldots \varphi_{j-1, m} \mu_{j, m} \ldots \mu_{j, l-1}, H_{j, l} / H_{j, l+1}$ to be a closed $\left(f_{1}, \ldots, f_{n-1}\right)$-stable subgroup of $H_{k, m} / H_{k, m+1}$. Again the induction hypothesis implies there exist positive integers $j_{1}, l_{1}$ such that $\mu_{j, l}, \varphi_{j, l}$ are isomorphisms if $j \geq j_{1}$ and $l \geq l_{1}$. It follows from above that $\alpha_{j, l}$ is also an isomorphism.

Similarly one shows there exist positive integers $j_{2}, l_{2}$ such that $\psi_{j, l}, \beta_{j, l}$ and $\nu_{j, l}$ are isomorphisms if $j \geq j_{2}$ and $l \geq l_{2}$.

Let $j_{0}=\max \left\{j_{1}, j_{2}\right\}$ and $l_{0}=\max \left\{l_{1}, l_{2}\right\}$.

We claim $C=H^{\left(j_{0}\right)} \cap \bigcap_{k=-l_{0}}^{l_{0}} f_{n}^{k}(E) \subseteq H$. If not, there exists an open subgroup $J$ of $G$ such that $J \supseteq H$ and $J \nsupseteq C$. Since $\cap_{k=-\infty}^{\infty} f_{n}^{k}(E)=e$ it follows from Lemma 2.1 that there exists an integer $p \geq l_{0}$ such that $J \nsupseteq H^{\left(j_{0}\right)} \cap$ $\bigcap_{k=-p}^{p} f_{n}^{k}(E)$ and $J \supseteq H^{\left(j_{0}\right)} \cap \cap_{k=-p-1}^{p+1} f_{n}^{k}(E)$. Either (a) or (b) below can occur:
(a)
$J \nsupseteq H^{\left(j_{0}\right)} \cap \bigcap_{k=-p}^{p} f_{n}^{k}(E) \quad$ and
$J \supseteq H^{\left(j_{0}\right)} \cap \bigcap_{k=-p}^{p+1} f_{n}^{k}(E)$
(b)
$J \nsupseteq H^{\left(j_{0}\right)} \cap \bigcap_{k=-p}^{p+1} f_{n}^{k}(E) \quad$ and
$J \supseteq H^{\left(j_{0}\right)} \cap \bigcap_{k-p-1}^{p+1} f_{n}^{k}(E)$. 
If (a) occurs we have $J^{\prime}=f_{n}^{p}(J) \nsupseteq H^{\left(j_{0}\right)} \cap \bigcap_{k=0}^{2 p} f_{n}^{k}(E)$ and $J^{\prime} \supseteq H^{\left(j_{0}\right)} \cap$ $\cap_{k=0}^{p+1} f_{n}^{k}(E) . J^{\prime}$ is an open and hence closed subgroup of $G$. We have the following sequence of mappings

$$
H_{0,2 p} / H_{0,2 p+1} \stackrel{\tau_{1}}{\longrightarrow} J \cap H_{0,2 p} / H_{0,2 p+1} \stackrel{\tau_{2}}{\longrightarrow} J \cap H_{j_{0}, 2 p} / H_{j_{0}, 2 p+1} \stackrel{\tau_{3}}{\longrightarrow} H_{j_{0}, 2 p} / H_{j_{0}, 2 p+1}
$$

where $\tau_{1}$ is the identity, $\tau_{2}$ is the natural map, and $\tau_{3}$ is an injection. $\tau_{1}$ is an isomorphism, $\tau_{2}$ is a monomorphism, and $\tau_{3}$ is a monomorphism which is not epic. Hence $\tau_{3} \tau_{2} \tau_{1}$ is not epic. But $\tau_{3} \tau_{2} \tau_{1}=\alpha_{j 0,2 p}$, contradicting the fact that $\alpha_{l, j}$ is an isomorphism if $l \geq l_{0}$ and $j \geq j_{0}$.

If (b) occurs we can, using an argument similar to the one above, contradict the fact that $\beta_{j, l}$ is an isomorphism for some $l$ and $j$ where $l \geq l_{0}$ and $j \geq j_{0}$.

Hence $C \subseteq H \subseteq H^{\left(j_{0}\right)}$.

$C$ is an open subgroup of $H^{\left(j_{0}\right)}$ and

$$
\cap\left\{f_{1}^{k_{1}} \ldots f_{n-1}^{k_{n-1}}\left(H^{\left(j_{0}\right)} \cap \bigcap_{k=-l_{0}}^{l_{0}} f_{n}^{k}(N)\right) \mid k_{1}, \ldots, k_{n-1} \text { integers }\right\}=C .
$$

By Proposition $2.1,\left(H^{\left(j_{0}\right)} / C ; f_{1}, \ldots, f_{n-1}\right)$ is of finite type. Since $H / C$ is a closed $\left(f_{1}, \ldots, f_{n-1}\right)$-stable subgroup of $H^{\left(j_{0}\right)} / C$, the induction hypothesis implies there exists an open subgroup $Q$ of $G$ such that

$$
\cap\left\{f_{1}^{k_{1}} \ldots f_{n-1}^{k_{n-1}}\left(H^{\left(j_{0}\right)} \cap Q\right) \mid k_{1}, \ldots, k_{n-1} \text { integers }\right\}=H .
$$

Since $H$ is stable, $\cap f_{1}^{k_{1}} \ldots f_{n}^{k_{n}}\left(H^{\left(j_{0}\right)} \cap Q\right)=H$. Let $R=N^{\left(j_{0}\right)} \cap Q$ an open subgroup of $G$. Then

$$
\begin{aligned}
\cap f_{1}^{k_{1}} \ldots f_{n}^{k_{n}}(R) & =\cap f_{1}^{k_{1}} \ldots f_{n}^{k_{n}}\left(N^{\left(j_{0}\right)}\right) \cap \cap f_{1}^{k_{1}} \ldots f_{n}^{k_{n}}(Q) \\
& =H^{\left(j_{0}\right)} \cap \cap f_{1}^{k_{1}} \ldots f_{n}^{k_{n}}(Q) \\
& =\cap f_{1}^{k_{1}} \ldots f_{n}^{k_{n}}\left(H^{\left(j_{0}\right)} \cap Q\right)=H .
\end{aligned}
$$

By Proposition 2.1, $\mathcal{M} / \mathbb{K}$ is finitely generated. (In the case $n=1,(H: C)$ $<\left(H^{\left(j_{0}\right)}: C\right)<\infty$. Since $\bigcap_{j=0}^{\infty} H^{(j)}=H$ there is a positive integer $t$ such that $H^{\left(j_{0}+t\right)}=H$. Thus $\mathcal{M}=\mathcal{L}\left(H^{\left(j_{0}+t\right)}\right)$ is finitely generated over $\mathcal{K}$. $)$

3. Limit groups of ordinary difference fields. Throughout this section $\mathscr{K}$ will be a difference field with underlying field $K$ and one transforming operator $\sigma$, and $\mathcal{L}$ will be a finitely generated extension of $\mathcal{K}$ whose underlying field $L$ is Galois over $K ; G$ will be the Galois group of $L / K$ and $f$ the automorphism of $G$ induced by $\sigma$.

Since $\mathcal{L} / \mathscr{K}$ is finitely generated, it follows from Proposition 2.1 that there is an open invariant subgroup $N$ of $G$ such that $\cap N_{k}=(e)$. As in the proof of Theorem 2.1, we have monomorphism $\varphi_{0}: N / N \cap N_{1} \rightarrow G / N$ and 


$$
\varphi_{i}: \bigcap_{k=0}^{i} N_{k} / \bigcap_{k=0}^{i+1} N_{k} \rightarrow \bigcap_{k=0}^{i-1} N_{k} / \bigcap_{k=0}^{i} N_{k} \quad \text { for } i=1,2, \ldots
$$

where $\varphi_{i}\left(g \cap_{k=0}^{i+1} N_{k}\right)=f^{-1}(g) \cap_{k=0}^{i} N_{k}$. Since all the above factor groups are finite, there is an $m$ such that $\varphi_{n}$ is an isomorphism if $n \geq m$.

Definition 3.1. The subgroup $\varphi_{0} \ldots \varphi_{m}\left(\cap_{k=0}^{m} N_{k} / \cap_{k=0}^{m+h} N_{k}\right)$ of $G / N$ is called the limit group of $G$ (or $\mathcal{L} / \mathscr{K}$ ) with respect to $N$, and we denote it by $l_{N}(G)$.

$l_{N}(G)$ is not independent of our choice of $N$, as the following example shows:

Example 3.1. Let $\ldots, t_{-1}, t_{0}, t_{1}, \ldots$ be a collection of elements algebraically independent over the field $C$ of complex numbers, and let $L=C\left(\ldots, t_{-1}, t_{0}\right.$, $\left.t_{1}, \ldots\right)$. Let $\sigma$ be the automorphism on $L$ which is the identity on $C$ and maps $t_{i}$ into $t_{i+1} . \mathcal{L}$ will be the difference field with underlying field $L$ and transforming operator $\sigma$. Define $\mathcal{K}$ to be the difference field with underlying field $K$ $=C\left(\ldots, x_{-1}, x_{0}, x_{1}, \ldots\right)$ where $x_{i}=t_{i}^{4}$ and transforming operator $\sigma \mid K$. Clearly $L / K$ is Galois.

Let $N$ be the subgroup of $G$, the Galois group of $L / K$, with fixed field $K\left(t_{0}\right)$. Since $K\left(t_{0}\right) / K$ is finite dimensional Galois, $N$ is an open invariant subgroup of G. Also $\cap N_{k}=(e)$ since the fixed field of $\cap N_{k}$ is $K\left(\cup_{k=-\infty}^{\infty} \sigma^{k}\left(K\left(t_{0}\right)\right)\right)=L$. $G / N$ is the Galois group of $K\left(t_{0}\right) / K$ and $\bigcap_{k=0}^{i} N_{k} / \bigcap_{k=0}^{i+1} N_{k}$ is the Galois group of $K\left(t_{0}, \ldots, t_{i+1}\right) / K\left(t_{0}, \ldots, t_{i}\right)$. By an order argument one easily sees that $l_{N}(G)=G / N . G / N$ is the cyclic group of order 4 .

Let $M$ be the subgroup of $G$ with fixed field $K\left(t_{0}, t_{1}^{2}\right)$. Again $M$ is an invarient, open subgroup of $G$ and $\cap M_{k}=(e)$. $\cap_{k=0}^{i} M_{k} / \cap_{k=0}^{i+1} M_{k}$ is the Galois group of $K\left(t_{0}, \ldots, t_{i+1}^{2}, t_{i+2}\right) / K\left(t_{0}, \ldots, t_{i}, t_{i+1}^{2}\right)$ which has dimension 4 for $i=1,2, \ldots$ Hence

$$
l_{M}(G)=M / M \cap M_{1}
$$

$M / M \cap M_{1}$ is the Galois group of $K\left(t_{0}, t_{i}, t_{2}^{2}\right) / K\left(t_{0}, t_{1}^{2}\right)$. Hence $l_{M}(G)$ is the Klein four group.

There are however many properties of limit groups which are independent of the choice of $N$. Theorem 3.1 will reflect these properties.

One should note that the order of a limit group of $\mathcal{L} / \mathcal{K}$ is the limit degree of the extension. (See [4, pp. 135-136].)

Proposition 3.1. If $N$ is an open invariant subgroup of $G$ such that $\cap N_{k}=(e)$ then for each pair of integers $s, t$ with $s \leq t, M=\cap_{k=s}^{t} N_{k}$ is an open invariant subgroup of $G$ with $\cap M_{k}=(e)$ such that $l_{M}(G) \cong l_{N}(G)$.

Proof. Choose $m$ sufficiently large so that $l_{N}(G) \cong \bigcap_{k=0}^{n} N_{k} / \cap_{k=0}^{n+1} N_{k}$ and $l_{M}(G) \cong \bigcap_{k=0}^{n} M_{k} / \cap_{k=0}^{n+1} M_{k}$ if $n \geq m$.

$$
l_{M}(G) \cong \bigcap_{k=s}^{m+t} N_{k} / \bigcap_{k=s}^{m+t+1} N_{k} \cong \bigcap_{k=0}^{m+t-s} N_{k} / \bigcap_{k=0}^{m+t-s+1} N_{k} \cong l_{N}(G)
$$


Proposition 3.2. If $N$ is an open invariant subgroup of $G$ such that $\cap N_{k}=(e)$ then, for each integer $r, l_{N}(G)$ is isomorphic to $H_{r}=\bigcap_{k=-\infty}^{r} N_{k} / \bigcap_{k=-\infty}^{r+1} N_{k}$.

Proof. Since $\mu: H_{r} \rightarrow H_{0}$ given by $\mu\left(g \cap_{k=-\infty}^{r} N_{k}\right)=f^{-r}(g) \cap_{k=-\infty}^{0} N_{k}$ is an isomorphism for each integer $r$, we may assume in the proof of Proposition 3.2 that $r=0$. Choose a nonnegative integer $n$ such that if $m \geq n$ then $l_{N}(G)$ $\cong \bigcap_{k=0}^{n} N_{k} / \bigcap_{k=0}^{n+1} N_{k}$. Consider the' monomorphism

$$
\varphi: H_{0} \rightarrow \bigcap_{k=0}^{n} N_{k} / \bigcap_{k=0}^{n+1} N_{k}
$$

given by $\varphi\left(g \cap_{k=-\infty}^{1} N_{k}\right)=f^{n}(g) \cap_{k=0}^{n+1} N_{k} . \cap_{k=-\infty}^{n} N_{k}$ is a closed invariant subgroup of $\cap_{k=0}^{n} N_{k} \cdot\left\{\bigcap_{k=-j}^{n} N_{k} / \bigcap_{k=-\infty}^{n} N_{k} \mid j=1,2, \ldots\right\}$ is a basis at the identity for $\bigcap_{k=0}^{n} N_{k} / \bigcap_{k=-\infty}^{n} N_{k}$. If $\varphi$ is not an isomorphism then $\left(\cap_{k=-\infty}^{n} N_{k}\right)\left(\cap_{k=0}^{n+1} N_{k}\right)$ is a proper open subgroup of $\bigcap_{k=0}^{n} N_{k}$. So, there exists a positive integer $j$ such that

$$
\left(\bigcap_{k=-\infty}^{n} N_{k}\right)\left(\bigcap_{k=0}^{n+1} N_{k}\right)\left(\bigcap_{k=-j}^{n} N_{k}\right)=\left(\bigcap_{k=0}^{n+1} N_{k}\right)\left(\bigcap_{k=-j}^{n} N_{k}\right) \subset \bigcap_{k=0}^{n} N_{k} .
$$

(1) implies the natural map

$$
\eta: \bigcap_{k=-j}^{n} N_{k} / \bigcap_{k=-j}^{n+1} N_{k} \rightarrow \bigcap_{k=0}^{n} N_{k} / \bigcap_{k=0}^{n+1} N_{k}
$$

is not epic. $\eta$ is one-to-one and by Proposition 3.1 both factor groups are isomorphic to $l_{N}(G)$ and thus are of the same order. Hence $\eta$ is epic, a contradiction.

Theorem 3.1. If $M, N$ are open invariant subgroups of $G$ such that $\cap M_{k}=\cap N_{k}$ $=(e)$ then $l_{M}(G)$ and $l_{N}(G)$ have equivalent composition series.

Proof. $M$ is an open subgroup of $G$ and $\left\{\cap_{k=-j} f^{k}(N) \mid j=0,1, \ldots\right\}$ is a decreasing sequence of closed subgroups of $G$ with intersection (e). Lemma 2.1 implies there is a positive integer $p$ such that $\cap_{k=-p}^{p} N_{k} \subseteq M$. Proposition 3.1 implies $l_{N}(G) \cong l_{N^{\prime}}(G)$ where $N^{\prime}=\bigcap_{k=-p}^{p} N_{k}$. Hence we may assume $N \subseteq M$.

There exists a positive integer $n$ such that $N \supseteq \cap_{k=-n}^{n} M_{k}$. We have the following sequence of open invariant subgroups of $G$.

$$
M \supseteq N \supseteq \bigcap_{k=-n}^{n} N_{k} \supseteq \bigcap_{k=-n}^{n} M_{k} \supseteq \bigcap_{k=-2 n}^{2 n} N_{k} \supseteq \ldots
$$

which gives rise to the following chain of closed invariant subgroups of $G$ :

$$
\bigcap_{k=-\infty}^{0} M_{k} \supseteq \bigcap_{k=-\infty}^{0} N_{k} \supseteq \bigcap_{k=-\infty}^{n} M_{k} \supseteq \bigcap_{k=-\infty}^{n} N_{k} \supseteq \bigcap_{k=-\infty}^{2 n} M_{k} \supseteq \ldots
$$

By refining the normal series

and

$$
\bigcap_{k=-\infty}^{0} M_{k} \supseteq \bigcap_{k=-\infty}^{0} N_{k} \supseteq \bigcap_{k=-\infty}^{1} N_{k} \supseteq \ldots \supseteq \bigcap_{k=-\infty}^{n} N_{k} \supseteq \bigcap_{k=-\infty}^{2 n} M_{k}
$$

$$
\bigcap_{k=-\infty}^{0} M_{k} \supseteq \bigcap_{k=-\infty}^{1} M_{k} \supseteq \ldots \supseteq \bigcap_{k=-\infty}^{2 n} M_{k}
$$


to composition series for $\bigcap_{k=-\infty}^{0} M_{k} / \bigcap_{k=-\infty}^{2 n} M_{k}$ we see that the factors of a composition series of $l_{N}(G) \cong \bigcap_{k=-\infty}^{0} N_{k} / \bigcap_{k=-\infty}^{1} N_{k}$ are contained among the factors of a composition series for $l_{M}(G) \cong \bigcap_{k=-\infty}^{j} M_{k} / \cap_{k=-\infty}^{j+1} M_{k}, j=0, \ldots$, $2 n-1$. Similarly one shows the factors of a composition series of $l_{M}(G)$ are contained among the factors of a composition series of $l_{N}(G)$.

To complete the proof we show that the number of repetitions of any factor in a composition series for $l_{M}(G)$ is equal to the number of repetitions of that factor in a composition series for $l_{N}(G)$.

Let $H$ be a factor of the two composition series and suppose it appears $a$ times in the factors of $l_{M}(G)$ and $b$ times among the factors of $l_{N}(G)$. For each positive integer $l$ a refinement of the series $\cap_{k=-\infty}^{0} M_{k} \supseteq \cap_{k=-\infty}^{l} M_{k} \supseteq \ldots \supseteq \cap_{k=-\infty}^{l n} M_{k}$ to correspond to a composition series for $B=\bigcap_{k=-\infty}^{0} M_{k} / \bigcap_{k=-\infty}^{l n} M_{k}$ contains $H$ lna times. By refining $\bigcap_{k=-\infty}^{0} M_{k} \supseteq \bigcap_{k=-\infty}^{0} N_{k} \supseteq \bigcap_{k=-\infty}^{1} N_{k} \supseteq \ldots \supseteq$ $\bigcap_{k=-\infty}^{(l-1) n} N_{k} \supseteq \bigcap_{k=-\infty}^{l n} M_{k}$ to determine a composition series for $B$ we get $(l-1) n b$ copies of $H$ among the factors determined by the subgroups of this composition series between $\cap_{k=-\infty}^{0} N_{k}$ and $\bigcap_{k=-\infty}^{(l-1) n} N_{k}$. Hence for each positive integer $l$, $(l-1) n b \leq \ln a$; thus $b \leq a$.

Similarly, using $\cap_{k=-\infty}^{0} N_{k} \supseteq \cap_{k=-\infty}^{n} M_{k} \supseteq \cap_{k=-\infty}^{l n} M_{k} \supseteq \bigcap_{k=-\infty}^{l n} N_{k}$ to represent a normal series for $\bigcap_{k=-\infty}^{0} N_{k} / \bigcap_{k=-\infty}^{l n} N_{k}, l=1,2, \ldots$, one shows $a \leq b$.

Theorem 3.2. Let $\mathcal{N} \mathscr{K}$ be an inversive subextension of $\mathcal{L} / \mathcal{K}$ with underlying field $M$ Galois over $K$, and let $H=G(\triangle M)$. Then, given any limit group $A$ of $G$ there exist limit groups $B, C$ of $H, G / H$ respectively and an imbedding $\alpha$ of $B$ into $A$ such that $A / \alpha(B) \cong C$.

Proof. Clearly $H$ is of finite type since $G$ is of finite type. By Theorem 2.1, $G / H$ is of finite type. Hence $H$ and $G / H$ have limit groups.

Choose an open invariant subgroup $N$ of $G$ such that $\cap N_{k}=e$. There exist nonnegative integers $n_{1}, n_{2}$ such that if $n \geq n_{1}$ then $l_{N}(G) \cong \bigcap_{k=0}^{n} N_{k} / \cap_{k=0}^{n+1} N_{k}$ and if $n \geq n_{2}$ then $l_{N \cap H}(H) \cong \bigcap_{k=0}^{n} f^{k}(N \cap H) / \cap_{k=0}^{n+1} f^{k}(N \cap H)$.

Choose an open invariant subgroup $E$ of $G$ such that $\cap E_{k}=H$ (Theorem 2.1). $\left\{\cap_{k=-j}^{j} N_{k} \mid j=0,1,2, \ldots\right\}$ is a decreasing sequence of closed subgroups of $G$ whose intersection is contained in $H$. By Lemma 2.1 there exists a positive integer $a$ such that $\cap_{k=-a}^{a} N_{k} \subseteq E . \cap_{k=0}^{2 a} N_{k} \subseteq E_{a}$ and $F=H\left(\cap_{k=0}^{2 a} N_{k}\right) \subseteq E_{a}$. Since $H$ is stable $H \subseteq \cap F_{k} \subseteq \cap E_{k}=H$. Hence there exists a positive integer $n_{3}$ such that if $J=\bigcap_{k=0}^{n_{3}} N_{k}$ then $\cap f^{k}(J H)=H$. Let $n_{0}=\max \left\{n_{1}, n_{2}, n_{3},\right\}$, $X=\bigcap_{k=0}^{n_{0}} N_{k}, Y=X \cap H$ and $Z=X H$. By Proposition 3.1, $l_{N}(G) \cong l_{X}(G)$. $l_{X}(G) \cong X / X \cap X_{1}$ and $l_{Y}(H) \cong Y / Y \cap Y_{1}$. There exists a nonnegative integer $m$ such that if $n \geq m$ then $l_{Z / H}(G / H) \cong \cap_{k=0}^{n} Z_{k} / \cap_{k=0}^{n+1} Z_{k}$.

For each positive integer $n$, the monomorphism $\varphi_{n}: \bigcap_{k=0}^{n} Z_{k} / \cap_{k=0}^{n+1} Z_{k} \rightarrow$ $\cap_{k=0}^{n-1} Z_{k} / \cap_{k=0}^{n} Z_{k}$ defined by $\varphi_{n}\left(g \cap_{k=0}^{n+1} Z_{k}\right)=f^{-1}(g) \cap_{k=0}^{n} Z_{k}$ is an isomorphism. To show $\varphi_{n}$ epic consider 


$$
D=\left[\bigcap_{k=-1}^{n-1} Z_{k}\right]\left[\bigcap_{k=0}^{n} Z_{k}\right]=\bigcap_{k=0}^{n-1} Z_{k} \cap\left(\bigcap_{k=-1}^{n-1} Z_{k}\right) Z_{n}
$$

Now,

$$
\begin{aligned}
\left(\bigcap_{k=-1}^{n-1} Z_{k}\right) Z_{n} & =\left(\bigcap_{k=-1}^{n-1} Z_{k}\right) X_{n} H \supseteq\left(\bigcap_{k=-1}^{n-1} X_{1}\right)\left(X_{n} \cap X_{n-1}\right) H \\
& =f^{n-1}\left(\left(\bigcap_{k=-n}^{0} X_{k}\right)\left(X \cap X_{1}\right) H\right)=Z_{n-1} .
\end{aligned}
$$

The last equality follows from the fact that since $l_{X}(G)=X / X \cap X_{1}$, the monomorphism $\psi: \cap_{k=0}^{n} X_{k} / \cap_{k=0}^{n+1} X_{k} \rightarrow X / X \cap X_{1}$ given by $\psi\left(g \cap_{k=0}^{n+1} X_{k}\right)$ $=f^{-n}(g)\left(X \cap X_{1}\right)$ is an isomorphism. Hence $D=\cap_{k=0}^{n-1} Z_{k}$ and $\varphi_{n}$ is an isomorphism for $n>0$, and thus $l_{Z / H}(G / H) \cong Z / Z \cap Z_{1}$.

Let $\alpha: Y / Y \cap Y_{1} \rightarrow X / X \cap X_{1}$ be the natural monomorphism. The image of $\alpha$ is $Y\left(X \cap X_{1}\right) / X \cap X_{1}$. We complete the proof by showing the natural map $\tau$ of $X / X \cap X_{1}$ modulo the image of $\alpha$, i.e. $X / Y\left(X \cap X_{1}\right)$, into $Z / Z \cap Z_{1}$ is an isomorphism. $\tau$ is onto since $X\left(Z \cap Z_{1}\right)=X H\left(Z \cap Z_{1}\right)=Z$. From the remark following Example 3.1 and Theorem XII of Chapter 5 in [4] it follows that the domain and range of $\tau$ have the same order. Hence $\tau$ is an isomorphism.

4. Benign extensions. Throughout this section $\mathcal{L}$ will be a difference field with one transforming operator, finitely generated over the difference field $\mathscr{K}$. The underlying extension of $\mathcal{L} / \mathscr{K}$ will be Galois with Galois group $G$ and induced automorphism $f$.

Definition 4.1. The extension $\mathcal{L} / \mathscr{K}$ is benign (or $(G, f)$ or simply $G$ is benign) if there exists an open invariant subgroup $N$ of $G$ such that $\cap N_{k}=(e)$ and $l_{N}(G)=G / N$.

Lemma 4.1. If $\mathcal{L} / \mathcal{K}$ is benign and $N$ is an open invariant subgroup of $G$ with $\cap N_{k}=(e)$ and $l_{N}(G)=G / N$, then for $n=1,2, \ldots$,

$$
l_{N}(G) \cong \bigcap_{k=-n}^{-1} N_{k} / \bigcap_{k=-n-1}^{-1} N_{k}
$$

Proof. Consider the map $\varphi: N_{-1} / N_{-1} \cap N_{-2} \rightarrow G / N$ given by $\varphi\left(g\left(N_{-1} \cap N_{-2}\right)\right)=f^{2}(g) N$. Clearly $\varphi$ is a monomorphism. To show $\varphi$ onto we show $G=N f^{2}\left(f^{-1}(N)\right)=N N_{1}$. Since $G / N \cong N / N \cap N_{1}$ under the map $\psi\left(g\left(N \cap N_{1}\right)\right)=f^{-1}(g) N . G=N N_{-1}$ and since $G=f(G), G=N_{1} N$. Thus $\varphi$ is an isomorphism.

We complete the proof by showing that for each positive integer $n$ the monomorphism

$$
\tau_{n}: \bigcap_{k=-n-1}^{-1} N_{k} / \bigcap_{k=-n-2}^{-1} N_{k} \rightarrow \bigcap_{k=-n}^{-1} N_{k} / \bigcap_{k=-n-1}^{-1} N_{k}
$$

defined by $\tau_{n}\left(g \cap_{k=-n-2}^{-1} N_{k}\right)=f(g) \cap_{k=-n-1}^{-1} N_{k}$ is an isomorphism. Since $l_{N}(G)$ $=G / N, \varphi_{n}: \bigcap_{k=0}^{n} N_{k} / \cap_{k=0}^{n+1} N_{k} \rightarrow \bigcap_{k=0}^{n-1} N_{k} / \bigcap_{k=0}^{n} N_{k}$ defined by $\varphi_{n}\left(g \cap_{k=0}^{n+1} N_{k}\right)$ $=f^{-1}(g) \cap_{k=0}^{n} N_{k}$ is an isomorphism. Hence, 


$$
\bigcap_{k=0}^{n-1} N_{k}=\left(\bigcap_{k=0}^{n} N_{k}\right)\left(\bigcap_{k=-1}^{n-1} N_{k}\right)
$$

and so

$$
\bigcap_{k=-n}^{-1} N_{k}=f^{-n}\left(\bigcap_{k=0}^{n-1} N_{k}\right)=\left(\bigcap_{k=-n}^{0} N_{k}\right)\left(\bigcap_{k=-n-1}^{-1} N_{k}\right) .
$$

Hence $\tau_{n}$ is epic.

Lemma 4.2. If $\mathcal{L} / \mathcal{K}$ is benign and $N$ is an open invariant subgroup of $G$ such that $\cap N_{k}=(e)$ and $l_{N}(G)=G / N$, then for each integer $l$ and each positive integer $n$, $G=\left(\bigcap_{k=l-n}^{l-1} N_{k} \cap \bigcap_{k=l+1}^{++n} N_{k}\right) N_{l}$.

Proof. Since $f$ is an automorphism, it suffices to prove Lemma 4.1 with $l=0$.

$$
\begin{aligned}
A & =\left(\bigcap_{k=-n}^{-1} N_{k} \cap \bigcap_{k=1}^{n} N_{k}\right) N \supseteq\left(\bigcap_{k=-n}^{-1} N_{k} \cap \bigcap_{k=1}^{n} N_{k}\right)\left(\bigcap_{k=0}^{n} N_{k}\right) \\
& =\left(\bigcap_{k=-n}^{-1} N_{k}\right)\left(\bigcap_{k=0}^{n} N_{k}\right) \cap \bigcap_{k=1}^{n} N_{k} .
\end{aligned}
$$

Consider the monomorphism $\varphi: \bigcap_{k=0}^{n-1} N_{k} / \cap_{k=0}^{2 n} N_{k} \rightarrow G / \bigcap_{k=0}^{n} N_{k}$ defined by $\varphi\left(g \cap_{k=0}^{2 n} N_{k}\right)=f^{-n}(g) \cap_{k=0}^{n} N_{k}$. Since $G$ is benign with $l_{N}(G)=G / N,(G$ : $\left.\bigcap_{k=0}^{n} N_{k}\right)=(n+1)\left|l_{N}(G)\right|$ and $\left(\bigcap_{k=0}^{n-1} N_{k}: \bigcap_{k=0}^{2 n} N_{k}\right)=(n+1)\left|l_{N}(G)\right|$. Hence $\varphi$ is an isomorphism and $G=\left(\cap_{k=0}^{n} N_{k}\right)\left(\cap_{k=-n}^{-1} N_{k}\right)$.

This implies $A \supseteq \cap_{k=1}^{n} N_{k}$, and since $A \supseteq N, A \supseteq N\left(\cap_{k=1}^{n} N_{k}\right)$.

Now, consider the monomorphism $\psi: \bigcap_{k=-n}^{-1} N_{k} / \bigcap_{k=-n-1}^{-1} N_{k} \rightarrow G / N$ given by $\psi\left(g \cap_{k=-n-1}^{-1} N_{k}\right)=f^{n+1}(g) N$. By Lemma 4.1 both of these groups are isomorphic to $l_{N}(G)$; hence $\psi$ is an isomorphism and $G=N\left(\cap_{k=1}^{n} N_{k}\right)$. It follows that $A=G$ and hence $G=\left(\bigcap_{k=-n}^{-1} N_{k} \cap \bigcap_{k=1}^{n} N_{k}\right) N$.

Corollary 4.1. For each integer $l$,

$$
G=N_{l}\left(\cap\left\{N_{k} \mid k \neq l\right\}\right)
$$

Proof. It suffices to give a proof when $l=0$. If $A=N\left(\cap\left\{N_{k} \mid k \neq 0\right\}\right) \subset G$ choose $g \in G, g \notin A$. Then, $g N \cap \cap\left\{N_{k} \mid k \neq 0\right\}=\varnothing . H_{m}=\bigcap_{k=-m}^{-1} N_{k} \cap$ $\bigcap_{k=1}^{m} N_{k}, m=1,2, \ldots$, is a decreasing sequence of closed subgroups of $G$ such that $\cap_{m=1}^{\infty} H_{m} \subseteq G \sim g N$. By Lemma 2.1 there exists $H_{m} \subseteq G \sim g N$. Hence $g \notin N\left(\cap_{k=-m}^{-1} N_{k} \cap \cap_{k=1}^{m} N_{k}\right)$, contradicting Lemma 4.2. .

Theorem 4.1. Let $N$ be an open invariant subgroup of $G$ such that $\cap N_{k}=(e)$ and $H$ the topological group $\times G / N_{k}$.

(a) The mapping $f^{*}: H \rightarrow H$ defined by $f^{*}\left(g_{k} N_{k}\right)_{k}=\left(f\left(g_{k-1}\right) N_{k}\right)_{k}$ is a (topological) automorphism of $H$.

(b) The mapping $\varphi: G \rightarrow H$ defined by $\varphi(g)=\left(g N_{k}\right)_{k}$ is a monomorphism of 
topological groups such that $\varphi f=f^{*} \varphi$.

(c) If $\varphi$ is an isomorphism $G$ is benign.

(d) If $G$ is benign and $l_{N}(G)=G / N$ then $\varphi$ is an isomorphism.

Proof. (a) $f^{*}$ is well defined, for if $\left(g_{k} N_{k}\right)_{k}=\left(h_{k} N_{k}\right)_{k}$ then $g_{k}^{-1} h_{k} \in N_{k}$ for each integer $k$, and so $f\left(g_{k-1}^{-1} h_{k-1}\right)=f\left(g_{k-1}^{-1}\right) f\left(h_{h-1}\right) \in N_{k}$ for each integer $k . f^{*}$ is clearly multiplicative. If $\left(g_{k} \cdot N_{k}\right)_{k} \in \operatorname{ker}\left(f^{*}\right)$ then, for each $k, f\left(g_{k-1}\right) \in N_{k}$, and so $g_{k-1} \in N_{k-1}$; so $f^{*}$ is one-to-one. If $y=\left(g_{k} N_{k}\right)_{k}$ then $f^{*}(x)=y$ where $x=\left(f^{-1}\left(g_{k+1}\right) N_{k}\right)_{k}$. For each integer $k$ let $\varepsilon_{k}$ be the projection map of $H$ onto $G / N_{k}$. For each $k, \varepsilon_{k} f^{*}=\psi \varepsilon_{k}$ where $\psi$ is the isomorphism $\psi: G / N_{k} \rightarrow G / N_{k+1}$ given by $\psi\left(g N_{k}\right)=f(g) N_{k+1} \cdot \varepsilon_{k}$ is continuous and $\psi$, as an isomorphism of finite Galois groups, is continuous. Hence, $f^{*}$ is continuous. As a topological product of finite Galois groups $H$ is compact and Hausdorff and it follows that $f^{*}$ is bicontinuous.

(b) That $\varphi$ is a monomorphism of topological groups follows from Number 3, p. 190 in [2]. If $g \in G, \varphi f(g)=\left(f(g) N_{k}\right)_{k}=f^{*}\left(g N_{k}\right)_{k}=f^{*} \varphi(g)$.

(c) For integers $i_{1}, \ldots, i_{n}$ let $\times_{k \neq i_{1}, \ldots, i_{n}} G / N_{k}$ denote the subgroup $\times H_{k}$ of $H$ where $H_{k}=G / N_{k}$ if $k \neq i_{j}, j=1, \ldots, n$, and $H_{k}$ is the identity subgroup of $G / N_{k}$ otherwise. Since $\varphi$ is an isomorphism $G / N \cong H / \varphi(N) . H / \times_{k \neq 0} N_{k}$ $\cong G / N$ and $\varphi(N) \subseteq \times_{k \neq 0} N_{k}$ so $\varphi(N)=\times_{k \neq 0} N_{k}$. For each nonnegative integer $t$,

$$
\bigcap_{l=0}^{t} N_{l} / \bigcap_{l=0}^{t+1} N_{l} \cong \underset{k \neq 0, \ldots, t}{\times} G / N_{k} / \underset{k \neq 0, \ldots, t+1}{\times} G / N_{k} \cong G / N_{t+1} \cong G / N .
$$

Hence $l_{N}(G)=G / N$ and $G$ is benign.

(d) It only remains to show $\varphi$ is onto. This follows from Corollary 4.1 and Number 3, p. 190 in [2].

Remark 4.1. If $\mathcal{L} / \mathcal{K}$ is benign it follows that the underlying extension $L / K$ is isomorphic to $\bigotimes_{k=-\infty}^{\infty} K\left(\sigma^{k}(a)\right)$ over $K$ where $\sigma$ is the transforming operator and $a$ is an element of $L$ such that $\mathcal{L}=\mathscr{K} \ll a \gg$. (See [2, pp. 190-191].)

Definition 4.2. Suppose $\mathcal{L} / \mathscr{K}$ is as described at the beginning of $\S 4$ except perhaps not finitely generated. An automorphism $g \in G$ is called a (difference) automorphism of $\mathcal{L} / \mathcal{K}$ if $f(g)=g$.

Proposition 4.1. Let $\mathcal{L}$ and $\mathcal{K}$ be as in Definition 4.2. $D=\{g \mid f(g)=g\}$ is $a$ closed subgroup of $G$.

Proof. Clearly $D$ is a subgroup of $G$. To show $D$ is closed, consider the map $\lambda: G \rightarrow G$ defined by $\lambda(g)=g^{-1} f(g)$. $\lambda$ is continuous since it is the composition of the continuous maps $\alpha, \beta$ : when $G_{\rightarrow}^{\alpha} G \times G_{\rightarrow}^{\beta} G, \alpha(g)=\left(g^{-1}, f(g)\right)$, and $\beta(g, h)$ $=g h . D=\lambda^{-1}(e)$ is therefore closed in $G$.

Remark 4.2. $D$ will be called the Galois group of $\mathcal{L} / \mathcal{K}$. The mapping $\lambda$ defined in Proposition 4.1 will play an important role in $\$ 5$. We again return to consider extensions $\mathcal{L} / \mathscr{K}$ which are finitely generated. 
Proposition 4.2. If $N$ is an open invariant subgroup of $G$ such that $\cap N_{k}=(e)$ then the natural homomorphism $\eta: D \rightarrow G / N$, where $D$ is the Galois group of $\mathcal{L} / \mathcal{K}$, is a monomorphism. (A consequence of Proposition 4.2 is that if $\mathcal{L} / \mathcal{K}$ is finitely generated $D$ is a finite group.) If $G$ is benign and $l_{N}(G)=G / N$ then $\eta$ is an isomorphism.

Proof. If $g \in \operatorname{ker}(\eta)$ then $g \in N$, so $g=f^{k}(g) \in f^{k}(N)$ for each integer $k$. Then, $g \in \cap N_{k}=(e)$.

Now, suppose $l_{N}(G)=G / N$. Let $g N \in G / N$ and $x=\left(f^{k}(g) N_{k}\right)_{k} \in \times G / N_{k}$. Using the notation of Theorem 4.1 we see $f^{*}(x)=x$ and if $h=\varphi^{-1}(x) \in G$, then $f(h)=f \varphi^{-1}(x)=\varphi^{-1} f^{*}(x)=\varphi^{-1}(x)=h$. Clearly $h N=g N$ so $\eta(h)=g N$.

Corollary 4.2. For each integer $k$, let $D_{k}=D$ and let $J$ be the topological group $\times D_{k}$. The mapping $f_{*}\left(\left(g_{k}\right)_{k}\right)=\left(g_{k-1}\right)_{k}$ is a (topological) automorphism. $G$ is benign if and only if there exists a (topological) isomorphism $\psi: G \rightarrow J$ such that $\psi f=f_{*} \psi$.

5. Universally compatible and monadic extensions. As usual $\mathcal{L}$ will be a difference field with underlying field $L$ and transforming operator $\sigma$. $\mathscr{K}$ will be a subfield with underlying field $K . L / K$ will be Galois, $G$ will denote the group of automorphisms of $L / K$ and $f$ the automorphism of $G$ induced by $\sigma$.

Definition 5.1. Let $\mathcal{A}$ by an arbitrary extension of a difference field $\mathcal{B}$. $A / \mathcal{B}$ is universally compatible if given an extension $C / \mathcal{B}$ there exist an extension $D / \mathcal{B}$ and difference monomorphisms $\varphi: A / \mathcal{B} \rightarrow D / \mathcal{B}$ and $\psi: C / \mathcal{B} \rightarrow D / \mathcal{B}$. We will say the pair $(G, f)$ or simply $G$ is universally compatible if and only if $\mathcal{L} / \mathscr{K}$ is universally compatible.

Let $\lambda$ be the continuous function of $G$ into itself defined by $\lambda(g)=g^{-1} f(g)$. (See Proposition 4.9.) Let $\mathcal{M} / \mathcal{K}$ be a subextension of $\mathcal{L} / \mathcal{K}$ such that the underlying extension $M$ of $\mathcal{C}$ is normal over $K$ and let $H=G(c M)$. From Theorem 1.1 we know there exists an isomorphism $\varphi: G^{\prime} \rightarrow G / H$, where $G^{\prime}$ is the Galois group of $M / K$, such that $\varphi f^{\prime}=h \varphi$ where $f^{\prime}$ is the automorphism induced on $G^{\prime}$ by $\sigma \mid M$ and $h$ is the automorphism induced on $G / H$ by $f$. If $\lambda^{\prime}$ is the map defined on $G^{\prime}$ into itself by $\lambda^{\prime}\left(g^{\prime}\right)=g^{\prime-1} f^{\prime}(g)$ and $\lambda^{*}$ is the mapping defined on $G / H$ into itself by $\lambda^{*}(g H)=(g H)^{-1} h(g H)=g^{-1} f(g) H=\lambda(g) H$, then $\varphi \lambda^{\prime}=\lambda^{*} \varphi$.

Theorem 5.1. $\mathcal{L} / \mathscr{K}$ is universally compatible if and only if $\lambda$ maps $G$ onto itself.

The proof of Theorem 5.1 is an application of Theorem XIII in Chapter 9 of [4].

Proposition 5.1. If $\mathcal{L} / \mathcal{K}$ is universally compatible and $\mathcal{M} / \mathcal{K}$ is a subextension of $\mathcal{L} / \mathcal{K}$ then $\mathcal{M} / \mathscr{K}$ is universally compatible.

Proposition 5.2. Let $\mathcal{M} / \mathcal{K}$ be a subextension of $\mathcal{L} / \mathcal{K}$ with Galois underlying extension. If $\mathcal{L} / \mathcal{M}$ and $\mathcal{M} \mathscr{K}$ are universally compatible then $\mathcal{L} / \mathscr{K}$ is universally compatible. 
Lemma 5.1. If $H$ is an open invariant subgroup of $G$ such that $f(H) \subseteq H$ then $H$ is stable.

Theorem 5.2. $\mathcal{L} / \mathcal{K}$ is universally compatible if and only if every subextension of $\mathcal{L} / \mathcal{K}$ whose underlying field is finite dimensional Galois over $K$ is universally compatible.

Proof. Suppose $\mathcal{L} / \mathcal{K}$ is not universally compatible. There exists $g \in G$ such that $g \notin \lambda(G)$. Since $G$ is compact Hausdorff and $\lambda$ continuous $g^{-1} \lambda(G)$ is a closed subset of $G$. $e \notin g^{-1} \lambda(G)$ and so there exists an open invariant subgroup $N$ of $G$ such that $g^{-1} \lambda(G) \cap N=\varnothing$. Let $H$ be maximal among all the open invariant subgroups $N$ such that $N \cap g^{-1} \lambda(G)=\varnothing$. We claim $H$ is stable. By Lemma 5.1 it is only necessary to show $f(H) \subseteq H$. If $f(H) \nsubseteq H$ then $H f(H)$ is an invariant open subgroup of $G$ properly containing $H$. Hence $g^{-1} \lambda(G)$ $\cap H f(H) \neq \varnothing$ and there exist $h \in G, a, b \in H$ such that $g^{-1} \lambda(h)=a f(b)$. Now $g^{-1} h^{-1} f(h) f\left(b^{-1}\right)=a$. Since $H$ is invariant there exists $c \in H$ such that $g^{-1} b^{-1}$ $=c g^{-1}$. We now have $c g^{-1} b h^{-1} f\left(h b^{-1}\right)=c g^{-1} \lambda\left(h b^{-1}\right)=a g^{-1} \lambda(h b)=c^{-1} a$ $\in g^{-1} \lambda(G) \cap H=\varnothing$, a contradiction. $\mathcal{L}(H) / \mathscr{K}$ is a finite dimensional Galois subextension of $\mathcal{L} / \mathcal{K}$.

There is no $h \in G$ such that $g H=\lambda(h) H$, for if $g H=\lambda(h) H, g^{-1} \lambda(h) \in H$ $\cap g^{-1} \lambda(G)$. Hence $\mathcal{L}(H) / \mathscr{K}$ is not universally compatible.

The converse follows from Proposition 5.1.

Definition 5.2. The core of $G$ is the intersection of all open invariant stable subgroups of $G$. We denote the core by $C(G)$.

Note that $C(G)$ is closed, invariant and stable and is the intersection of all open stable subgroups of $G$. Further, $\mathcal{L}(C(G))$ is the core of the difference field extension $\mathcal{L} / \mathcal{K}$. (See $[4$, p. 215].)

Proposition 5.3. If $G$ is of finite type and $C(G)=(e)$ then $|G|<\infty$.

Proof. Let $\delta$ be the set of invariant, open, stable subgroups of $G$. Choose an open subgroup $N$ of $G$ such that $\cap N_{k}=(e)$. Since $G$ is compact and the elements of $\delta$ are closed on $G$ there exist $H^{(1)}, \ldots, H^{(n)} \in \mathcal{S}$ such that $H=\bigcap_{i=1}^{n} H^{(i)} \subseteq N . H$ is stable; hence $(e)=C(G) \subseteq H \subseteq \cap N_{k}=(e)$. Since $H$ is open in $G, \infty>(G: H)=|G|$.

Corollary 5.1. If $G$ is of finite type then $(G: C(G))<\infty$.

Proof. By Theorem 2.1, $G / C(G)$ is of finite type and clearly the core of $G / C(G)$ with respect to the automorphism induced on $G / C(G)$ by $f$ is $C(G)$.

Corollary 5.2. If $G$ is of finite type then $C(C(G))=C(G)$.

Proof. If $H$ is an open invariant subgroup of $C(G)$ then $(G: H)=(G: C(G))$ $\cdot(C(G): H)<\infty$. Hence $H=C(G)$. 
Corollary 5.3. $C(C(G))=C(G)$.

Proof. Suppose $C=C(G)$ has an open, invariant, stable, proper subgroup $H$. There exists an open invariant subgroup $N$ of $G$ such that $F=N \cap C \subseteq H$. Since $H$ is stable, $F_{k} \subseteq H$ for each integer $k$. Let $E^{\prime}$ be the subgroup of $H$ generated by $\cup F_{k}$. $E^{\prime}$ is stable and an invariant subgroup of $G$. Let $E$ be the closure of $E^{\prime}$ in $G$. It is easy to see that the closure of a stable group is stable. Hence $E$ is a closed, invariant, stable subgroup of $G$ contained in $H$ and $(C: E)<\infty$. Consider $G / E, C(G / E)=C / E \neq E$. By replacing $G$ by $G / E$ and $f$ by the automorphism induced on $G / E$ by $f$, we have reduced to the case where $C$ is a nontrivial finite subgroup of $G$.

Under this reduction $(e)$ is an open subgroup of $C$. There is an open invariant subgroup $A$ of $G$ such that $A \cap C=(e)$. Let $P=\cap A_{k}$, a closed invariant stable subgroup of $G . G / P$ is of finite type.

We claim $C(G / P)=C P / P$. Clearly $C P / P \subseteq C(G / P)$. If $C P=G$ then $G / P$ $=C P / P \cong C / C \cap P=C \subseteq(e)$. Hence $(G: P)<\infty$ (i.e. $P$ is open in $G$ ). Thus $P \supseteq C$ so $(e)=P \cap C=C$ contradicting the fact $C \neq(e)$. Hence we may assume there is a $g \in G$ with $g \notin C P$. If $g$ is such a point then $g P \cap C=\varnothing$. From the definition of $C$ and the fact that $G$ is compact it follows that there exist a finite number of open stable subgroups, $N^{(1)}, \ldots, N^{(l)}$, of $G$ such that $\varnothing=g P \cap \bigcap_{i=1}^{l} N^{(i)}$. If $B=\bigcap_{i=1}^{l} N^{(i)}$ then $B P / P$ is an open, invariant, stable subgroup of $G / P$ which does not contain $g P$. Hence, $C P / P \supseteq C(G / P)$.

We now have $G / P$ of finite type with core $C P / P \cong C / C \cap P=C$ which is finite. $P / P$ is an open invariant stable subgroup of $C(G / P)$, contradicting Corollary 5.2 .

Theorem 5.3. $\mathcal{L} / \mathscr{K}$ is universally compatible if and only if $\mathcal{L}(C) / \mathscr{K}, C=C(G)$, is universally compatible.

Proof. By Corollary 5.3, $\mathcal{L} / \mathcal{L}(C)$ has no finite dimensional subextensions and hence $\mathcal{L} / \mathcal{L}(C)$ is universally compatible (Theorem 5.2). Theorem 5.3 then follows from Proposition 5.2.

A difference field extension $A / B$ is monadic $[4, p .196]$ if the maximal number of difference isomorphisms of $A / B$ into any extension of $B$ is one. Since $L / K$ is Galois, $\mathcal{L} / \mathcal{K}$ is monadic if and only if the difference Galois group $D$ $=\{g \in G \mid f(g)=g\}$ of $\mathcal{L} / \mathscr{K}$ is the identity group. As usual we will say $(G, f)$ or simply $G$ is monadic if and only if $\mathcal{L} / \mathscr{K}$ is monadic.

Theorem 5.4. $\mathcal{L} / \mathcal{K}$ is monadic if and only if $\lambda$ is one-to-one on $G .(\lambda(g)$ $\left.=g^{-1} f(g).\right)$

Proposition 5.4. If $\mathcal{L} / \mathscr{K}$ is monadic and $\mathcal{M} \mathscr{K}$ is a subextension of $\mathcal{L} / \mathscr{K}$ then $\mathcal{L} / \mathrm{Cl}$ is monadic.

Proof. From Theorem 5.4, this proposition is equivalent to showing if $\lambda$ is oneto-one on $G$ then $\lambda$ is one-to-one on any subgroup. 
Proposition 5.5. Let $\mathcal{L} / \mathcal{K}$ be finitely generated. $\mathcal{L} / \mathcal{K}$ is monadic if and only if $\mathcal{L} / \mathcal{M}$ is monadic for every subextension $\mathcal{M} \mathscr{K}$ of $\mathcal{L} / \mathscr{K}$ where $[L: M]<\infty, M$ being the underlying field of $M$.

Proof. If $\mathcal{L} / \mathscr{K}$ is not monadic then $D \neq(e)$. By Proposition 4.2, $D$ is finite and $[L: L(D)]<\infty$. Clearly $\mathcal{L} / \mathcal{L}(D)$ is not monadic.

The converse follows from Proposition 5.4.

Lemma 5.2. Let $\mathcal{M} / \mathcal{K}$ be a subextension of $\mathcal{L} / \mathcal{K}$ such that the underlying extension of $\mathcal{M} / \mathcal{K}$ is Galois. If $\mathcal{L} / \mathscr{K}$ is monadic and $\mathcal{L} / \mathcal{M}$ is universally compatible then $M / \mathcal{K}$ is monadic.

Proof. Let $H=G(c M)$. $\lambda$ is one-to-one on $G$ and maps $H$ onto itself. We want to show $\lambda^{\prime}$ is one-to-one on $G / H\left(\lambda^{\prime}(g H)=\lambda(g) H\right)$ or equivalently if $g \in G$ and $\lambda(g) \in H$ then $g \in H$. If $\lambda(g) \in H$ there exists $h \in H$ such that $\lambda(\dot{h})=\lambda(g)$. Hence, $g=h$.

Theorem 5.5. If $\mathcal{L} / \mathcal{K}$ is monadic and $\mathcal{L}(C) / \mathcal{K}$ is finitely generated where $C=C(G)$ then $\mathcal{L} / \mathcal{K}$ is universally compatible.

Proof. $\mathcal{L} / \mathcal{L}(C)$ is universally compatible. By Lemma 5.2, $\mathcal{L}(C) / \mathscr{K}$ is monadic. Hence, $\lambda^{\prime}: G / C \rightarrow G / C$ is onto. Hence $\mathcal{L}(C) / \mathscr{K}$ is universally compatible. By Theorem 5.3, $\mathcal{L} / \mathscr{K}$ is universally compatible.

Example 5.1. Theorem 5.5 implies that if $\mathcal{L} / \mathcal{K}$ is finitely generated and monadic then $\mathcal{L} / \mathcal{K}$ is universally compatible. We now construct an example of a monadic extension which is not universally compatible.

Let $\omega_{1}=-1$ and for $n=2,3, \ldots$ let $\omega_{n}$ be the primitive $2^{n}$ th root of unity which satisfies $x^{2}-\omega_{n-1}=0$. For $n=2,3, \ldots, \omega_{n-1} \omega_{n}$ is a primitive $2^{n}$ th root of unity. If $Q$ is the field of rational numbers we can define inductively on $Q\left(\omega_{n}\right)$ an automorphism $\sigma_{n}$ such that $\sigma_{n}\left(\omega_{n}\right)=\omega_{n-1} \omega_{n}$ and $\sigma_{n} \mid Q\left(\omega_{n-1}\right)=\sigma_{n-1}$. Let $K$ be $\cup_{n=1}^{\infty} Q\left(\omega_{n}\right)$ and $\sigma$ the automorphism defined on $K$ by the $\sigma_{n}$. Denote by $\mathscr{K}$ the difference field $(K, \sigma)$.

Let $\xi_{1}$ be a root of $x^{2}-5$. $\xi_{1} \notin K$, otherwise $\xi_{1} \in Q\left(\omega_{n}\right)$ for some $n$ and $\xi_{1} \in Q(\alpha)$ where $\alpha$ is a primitive fifth root of unity. (See [6, pp. 203-208].) $K \cap Q\left(\xi_{1}\right)=Q$ and $K / Q$ and $Q\left(\xi_{1}\right) / Q$ are normal extensions, $K$ and $Q\left(\xi_{1}\right)$ are linearly disjoint over $Q$, and there exists an automorphism $\tau$ of $Q\left(\xi_{1}\right) / Q$ such that $\tau\left(\xi_{1}\right)=\xi_{1}$. Hence there exists an automorphism $\sigma^{(1)}$ of $K\left(\xi_{1}\right)$ such that $\sigma^{(1)}\left(\xi_{1}\right)$ $=\xi_{1}$ and $\sigma^{(1)}$ extends $\sigma$. (See [3, pp. 12-15].) Let $\mathcal{L}_{1}$ be the difference field $\left(L_{1}, \sigma^{(1)}\right)$ where $L_{1}=K\left(\xi_{1}\right) . \mathcal{L}_{1} / \mathscr{K}$ is not universally compatible since it is finitely generated and not monadic. In particular $\varphi$ defined by $\varphi\left(\xi_{1}\right)=-\xi_{1}$ is a nontrivial difference automorphism of $\mathcal{L}_{1} / \mathscr{K}$. By Theorem 5.2 any extension of $\mathcal{L}_{1}$ is not universally compatible over $\mathscr{K}$.

We inductively define a sequence of difference fields $\left\{\mathcal{L}_{n}\right\}$ such that $\mathcal{L}_{n}$ is an extension of $\mathcal{L}_{n-1}, \mathcal{L}_{n}$ has underlying field $L\left(\xi_{n}\right)$ where $\xi_{n}$ is a root of $x^{2^{n}}-5$ and 
$\xi_{n}^{2}=\xi_{n-1}$, and $\mathcal{L}_{n}$ has transforming operator $\sigma^{(n)}$ where $\sigma^{(n)}\left(\xi_{n}\right)=\omega_{n}^{\alpha} \xi_{n}$ where $\alpha$ is 2 or $2^{n-1}+2$.

Let $\mathcal{L}$ be the difference field which is the union of the $\mathcal{L}_{n} . \mathcal{L} / \mathcal{K}$ is Galois and by our above remark is not universally compatible. We now show $\mathcal{L} / \mathcal{K}$ is monadic. If $\varphi$ is a (difference) automorphism of $\mathcal{L} / \mathcal{K}$ then $\varphi \mid \mathcal{L}_{n}$ is an automorphism of $\mathcal{L}_{n} / \mathscr{K}$ for each $n$. Let $\varphi\left(\xi_{n}\right)=\omega_{n}^{j} \xi_{n}$ where $j \in\left\{1,2, \ldots, 2^{n}\right\}$. $\varphi \sigma^{(n)}\left(\xi_{n}\right)=\varphi\left(\omega_{n}^{\alpha} \xi_{n}\right)=\omega_{n}^{\alpha} \omega_{n}^{j} \xi_{n}$ where $\alpha=2$ or $2^{n-1}+2$. $\sigma^{(n)} \varphi\left(\xi_{n}\right)=\sigma^{(n)}\left(\omega_{n}^{j} \xi_{n}\right)$ $=\left(\omega_{n-1} \omega_{n}\right)^{j} \omega_{n}^{\alpha} \xi_{n}$. Since $\varphi \sigma^{(n)}=\sigma^{(n)} \varphi$ we have $\omega_{n}^{\alpha+j}=\omega_{n}^{\alpha+3 j}$ and $\omega_{n}^{2 j}=1$. Hence, $\varphi\left(\xi_{n-1}\right)=\varphi\left(\xi_{n}^{2}\right)=\left(\omega_{n}^{j} \xi_{n}\right)^{2}=\xi_{n-1}$ and $\varphi \mid \mathcal{L}_{n-1}$ is the identity. Since $n$ is arbitrary, $\varphi$ is the identity on $\mathcal{L}$.

Theorem 5.6. Let $\mathcal{M} \mathscr{K}$ be an inversive subextension of $\mathcal{L} / \mathcal{K}$ with underlying extension of $\mathcal{M} \mathscr{K}$ Galois. Then:

(1) The difference Galois group $D_{2}$ of $\mathcal{L} / \mathcal{M}$ is a closed invariant stable subgroup of the difference Galois group, $D_{1}$, of $\mathcal{L} / \mathcal{K}$.

(2) There exists a natural monomorphism $\psi: D_{1} / D_{2} \rightarrow D_{3}$, where $D_{3}$ is the difference Galois group of $M / \mathscr{K}$. If $\mathcal{L} / \mathcal{M}$ is universally compatible then $\psi$ is an isomorphism.

(3) If $\mathcal{L} / \mathcal{K}$ is universally compatible and $\psi$ is an isomorphism then $\mathcal{L} / \mathcal{M}$ is universally compatible.

Proof. (1) Let $H=G(\triangle M) . \quad H$ is a closed invariant subgroup of $G . D_{2}$ $=D_{1} \cap H$. Hence (1).

(2) We identify $D_{3}$ with the subgroups of $G / H$ consisting of the elements $g H$ for which $\lambda(g) \in H$. If $\varphi: G \rightarrow G / H$ is the natural homomorphism and $g \in D_{1}$ then $\lambda(g)=e \in H$. Hence $\varphi \mid D_{1}$ is a homomorphism of $D_{1}$ into $D_{3}$ and its kernel is $D_{1} \cap H=D_{2}$. Let $\psi$ be the monomorphism $\psi: D_{1} / D_{2} \rightarrow D_{3}$ given by $\varphi . \psi$ is continuous and a closed map. If $\mathcal{L} / \mathcal{M}$ is universally compatible, so $\lambda(H)=H$ and $g H \in D_{3}$, then $\lambda(g) \in H$ and there exists $h \in H$ such that $\lambda(h)=\lambda(g)$. Thus $\lambda\left(g h^{-1}\right)=e$ and $g h^{-1} \in D_{1} \cdot \psi\left(g h^{-1} D_{2}\right)=g H$. Thus $\psi$ is an isomorphism of topological groups.

(3) Suppose $\psi$ is an isomorphism and $\lambda(G)=G$ (i.e., $\mathcal{L} / \mathcal{K}$ is universally compatible). Let $h \in H$. There exists a $g \in G$ such that $\lambda(g)=h$. $g H \in D_{3}$. Since $\psi$ is an isomorphism there exists a $g_{1} \in D_{1}$ such that $g_{1}=g h_{1}$ for some $h_{1} \in H \cdot g h_{1}=g_{1}=f\left(g_{1}\right)=f\left(g h_{1}\right)=g h f\left(h_{1}\right)$, so $\lambda\left(h_{1}^{-1}\right)=h$. Hence $\lambda(H)=H$.

Babbitt's Lemma. (See $[1, p .67]$.) If $G$ is of finite type, $C(G)=G$ and $G$ has no closed invariant stable subgroups $H$ such that the order of a limit group of $H$ is different from one or the order of a limit group of $G$, then $G$ is benign.

Proof. Let $\delta$ be the set of all open invariant subgroups $N^{\prime}$ of $G$ such that $\cap N_{k}^{\prime}=(e)$ and $l_{N^{\prime}}(G) \cong N^{\prime} / N^{\prime} \cap N_{1}^{\prime}, \delta \neq \varnothing$, for if $A$ is an open invariant subgroup of $G$ with $\cap A_{k}=e$ and $l_{A}(G) \cong \bigcap_{k=0}^{n} A_{k} / \bigcap_{k=0}^{n+1} A_{k}$ then $N^{\prime}=$ $\bigcap_{k=0}^{n} A_{k}$ is in $\delta$. Choose $N \in \delta$ whose index in $G$ is minimal. If the monomor- 
phism $\varphi: N / N \cap N_{1} \rightarrow G / N$ given by $\varphi\left(g N \cap N_{1}\right)=f^{-1}(g) N$ is onto we are done, so we assume $F=N N_{-1} \subset G$. If $F=N$ then, by Lemma 5.1, $N$ is stable. Since $(G: N)<\infty, G$ and $N$ have limit groups of equal order. Hence $N=G$, contradicting $F \neq G$. Thus, we also assume $N \subset F$.

Let $H=\cap F_{k}$ a closed invariant stable subgroup of $G$. The limit group of $H$ is (e); otherwise the order of a limit group of $G$ is equal to the order of a limit group of $H$ and, by Theorems 3.1 and 3.2, (e) is the limit group of $G / H$ which implies $(G: H)<\infty$. Since $C(G)=G, H=G$, a contradiction.

By Theorems 3.1 and 3.2, limit groups of $G$ and $G / H$ have the same order. $F / H$ and hence $F$ defines a limit group of $G / H$; thus $\left(F: F \cap F_{1}\right) \geq\left(N: N \cap N_{1}\right)$ since $l_{N}(G)=N / N \cap N_{1}$. Now

$$
\begin{aligned}
\left(G: F \cap F_{1}\right) & =(G: F)\left(F: F \cap F_{1}\right) \geq(G: F)\left(N: N \cap N_{1}\right) \\
& =\left(G: N N_{1}\right)\left(N N_{1}: N\right)=(G: N) .
\end{aligned}
$$

Since $N \subseteq F \cap F_{1}$ we also have $\left(G: F \cap F_{1}\right) \leq(F: N)$. Hence $N=F \cap F_{1}$ and $\cap F_{k}=(e)$ so $F$ defines a limit group of $G . \varphi$ maps $N / N \cap N_{1}$ onto $N N_{-1} / N=F / F \cap F_{1}$. Since $\left|l_{N}(G)\right|=\left|l_{F}(G)\right|, l_{F}(G)=F / F \cap F_{1}$. However, $(G: F)<(G: N)$, contradicting the minimality of $(G: N)$.

Theorem 5.7. If $G$ is of finite type and universally compatible then $D$ $=\{g \in G \mid f(g)=g\}$, the difference Galois group of $\mathcal{L} / \mathscr{K}$, and any limit groups of $G$ have equivalent composition series.

Proof. If the order of a limit group of $G$ is one then $G$ is finite. Since $G$ is finite and universally compatible $D=(e)$ and the theorem follows.

Assume the theorem true for all groups $G$ with limit groups of order less than $n, n \geq 1$, and let $G$ have limit groups of order $n$. By Theorem 3.2 there exist limit groups $A, B, E$ of $G, C(G)$ and $G / C(G)$ respectively and an imbedding $\varphi$ of $B$ into $A$ such that $A / \varphi(B) \cong E$. By Theorems 5.3 and 5.6 and Corollary 5.3, $D / D_{1}$ $\cong D_{2}$ where $D, D_{1}, D_{2}$ are the difference Galois groups of $G, C(G)$ and $G / C(G)$ respectively. Since $G / C(G)$ is universally compatible and $(G: C(G))<\infty$, $D_{2}=(e)$. Also $E=(e)$. Hence, $D=D_{1}$ and $A \cong B$. We can assume, therefore, that $G=C(G)$.

If $G$ has no invariant, closed stable subgroups $H$ such that the order of a limit group of $H$ is different from one or $n$ then by Babbitt's Lemma $G$ is benign. By Proposition 4.2, $G$ has a limit group isomorphic to $D$ and the theorem follows.

Suppose that $H$ is a closed invariant stable subgroup of $G$ with a limit group of order $k$ where $1<k<n$. Since $H$ is of finite type $C(H)$ is an open, invariant, stable subgroup of $H$. There exists an open invariant subgroup $N$ of $G$ such that $N \cap H \subseteq C^{\prime}(H) .(H: N \cap H)<\infty$ and $N \cap H$ is an invariant subgroup of $G$. The group generated by $\cup f^{k}(N \cap H)$ is an invariant stable subgroup of $G$. The closure $M$ of this group in $G$ is a closed invariant stable subgroup of $G$ contained 
in $C(H)$. Hence $M=C(H)$ and $C(H)$ is an invariant subgroup of $G$.

The order of any limit group of $C(H)$ is $k$. There exist limit groups $X, Y, Z$ of $G, C(H)$ and $G / C(H)$ respectively and an imbedding $\psi: Y \rightarrow X$ such that $X / \psi(Y) \cong Z$. Since $C(H)$ is universally compatible, $D / D_{3} \cong D_{4}$ where $D_{3}, D_{4}$ are the difference Galois groups of $C(H)$ and $G / C(H)$ respectively. By induction $D_{4}$ and $Z$ have equivalent composition series as do $D_{3}$ and $\psi(Y)$ and thus so do $D$ and $X$.

Corollary 5.4. If $G$ is monadic and of finite type then $G$ is finite.

Proof. $D=(e)$. By Theorem 5.5, $G$ is universally compatible and by the above theorem its finite groups are of order one. Hence $G$ is finite.

Example 5.2. We now give an example of a finitely generated extension $\mathcal{L} / \mathcal{K}$ which is not benign and whose core, $\mathcal{L}(C(G))$, is $\mathscr{K}$.

Let $\mathcal{K}$ be the difference field whose underlying field is $C(z)$ where $C$ is the complex numbers and $z$ is transcendental over $C$ and whose transforming operator is the automorphism $\sigma$ of $C(z)$ given by $\sigma(a)=a$ if $a \in C$ and $\sigma(z)=z+1$. Choose elements $u_{k}, v_{k}, w_{k}$ for $k=0, \pm 1, \ldots$ in the algebraic closure of $K$ such that $u_{k}^{2}=z+k, v_{k}^{2}=u_{k}+u_{k+1}$ and $w_{k}^{2}=u_{k}-u_{k-1}$ for each integer $k$. Let $L=K\left(\left\{u_{k}, v_{k} \mid k=0, \pm 1, \ldots\right\}\right)$ and let $\sigma$ be the automorphism of $L$ which extends $\sigma$ on $K$ and such that $\sigma\left(v_{k}\right)=v_{k+1}$ and $\sigma\left(w_{k}\right)=w_{k+1}$ for each integer $k$. Let $\mathcal{L}=(L, \sigma)$.

$\mathcal{L} / \mathscr{K}$ has core $\mathscr{K}$ and hence is universally compatible. (See [4, pp. 306-307].) $\mathcal{L} / \mathcal{K}$ is finitely generated; in fact $\mathcal{L}=\mathscr{K} \ll v_{0}, w_{0} \gg$. By Theorem 5.7 the order of $D$, the difference Galois group of $\mathcal{L} / \mathcal{K}$, is equal to the order of any limit group of $G$, the Galois group of $L / K$, which is equal to the limit degree of $\mathcal{L} / \mathcal{K}$. The limit degree of $\mathcal{L} / \mathcal{K}$ is 8 . (See [4, p. 135].)

$D$ consists of the automorphism $\varphi_{j, k}, \psi_{j, k}, j=1,2, k=1,2$, defined by $\varphi_{j, k}\left(v_{n}\right)=(-1)^{j} v_{n}, \varphi_{j, k}\left(w_{n}\right)=(-1)^{k} w_{n}, \psi_{j, k}\left(v_{n}\right)=(-1)^{j} i v_{n}$ and $\psi_{j, k}\left(w_{n}\right)=(-1)^{j} i w_{n}$ where $i^{2}=-1$. To see this let $\mathcal{M}=\mathscr{K} \ll u_{0} \gg$ a benign limit degree two extension of $\mathscr{K}$. The difference Galois group of $\mathcal{L} / \mathscr{K}$ consists of two elements, the identity, $\varphi$, and $\psi$ where $\psi\left(u_{n}\right)=-u_{n}$ for all $n$. $\mathcal{L} / \mathcal{C}$ is benign and hence universally compatible. By Theorem 5.6 every element in $D$ is an extension of either $\varphi$ or $\psi$. There are four possible extensions of each and they are given by $\varphi_{j, k}, \psi_{j, k}, j, k=1,2$. Since $|D|=8, D$ must consist of exactly these automorphisms. Corollary 4.2 implies that if $\mathcal{L} / \mathcal{K}$ is benign then $G \cong \times_{k=-\infty}^{\infty} D_{k}$ where $D_{k}=D$ for all $k$. Since $D$ is abelian, $G$ would also be abelian. To show $\mathcal{L} / \mathcal{K}$ not benign we construct an isomorphism $\tau$ of $L / K$ such that $\tau \varphi_{3}=\varphi_{3} \tau$.

Let $\rho$ be an automorphism of $K\left(\ldots, u_{-1}, u_{0}, u_{1}, \ldots\right) / K$ defined by $\rho\left(u_{2 n}\right)$ $=u_{2 n}, \rho\left(u_{2 n+1}\right)=-u_{2 n+1}$ for each integer $n$. Extend $\rho$ to an automorphism $\tau$ of $L / K$. If $\tau\left(v_{0}\right)=a$ then $a^{2}=\tau\left(v_{0}^{2}\right)=\tau\left(u_{0}+u_{1}\right)=u_{0}-u_{1}$, so $u= \pm w_{0}$. Now $\varphi_{3} \tau\left(v_{0}\right)=\varphi_{3}\left( \pm w_{0}\right)= \pm w_{0}$, but $\tau \varphi_{3}\left(v_{0}\right)=\tau\left(-v_{0}\right)=\mp w_{0}$. 


\section{BIBLIOGRAPHY}

1. A. E. Babbitt, Jr., Finitely generated pathological extensions of difference fields, Trans. Amer. Math. Soc. 102 (1962), 63-81. MR 24 \# A3160.

2. N. Bourbaki, Eléments de mathématiques. XI. I: Les structures fondamentales de lanalyse. Livre II: Algèbre. Chaps. IV, V, Actualités Sci. Indust., no. 1102, Hermann, Paris, 1950. MR 12, 6.

3.—- Eléments de mathématique. Part I. Les structures fondamentales de Tanalyse. Livre III: Topologie générale. Chap. I, Actualités Sci. Indust., no. 858, Hermann, Paris, 1940. MR 3, 55.

4. R. M. Cohn, Difference algebra, Interscience Tracts in Pure and Appl. Math., no. 17, Interscience, New York, 1965. MR 34 \# 5812.

5. N. Jacobson, Lectures in abstract algebra. Vol. 3: Theory of fields and Galois theory, Van Nostrand, Princeton, N. J., 1964. MR 30 \# 3087.

6. S. Lang, Algebra, Addison-Wesley, Reading, Mass., 1967. MR 33 \#5416.

7. D. Montgomery and L. Zippin, Topological transformation groups, Interscience Tracts in Pure and Appl. Math., no. 1, Interscience, New York, 1964.

8. L. S. Pontrjagin, Continuous groups, GITTL, Moscow, 1938; English transl., Topological groups, Princeton Math. Series, vol. 2, Princeton Univ. Press, Princeton, N. J., 1939. MR 1, 44.

Department of Mathematics, Lafayette College, Easton, Pennsylvania 18042 